Lineabilidade de Conjuntos em Espaços de Funções e de Zeros de Polinômios

Geraldo Perlino Júnior

\title{
DISSERTAÇÃO APRESENTADA
}

AO

INSTITUTO DE MATEMÁTICA E ESTATÍSTICA

DA

UNIVERSIDADE DE SÃO PAULO

PARA

OBTENÇÃO DO GRAU DE MESTRE

EM

MATEMÁTICA

Área de Concentração:Análise

Orientador: Profa. Dra. Mary Lilian Lourenço

São Paulo, 19 de agosto de 2005. 


\title{
Lineabilidade de Conjuntos em Espaços de Funções e de Zeros de Polinômios
}

\author{
Este exemplar corresponde à redação \\ final da dissertação devidamente corrigida \\ e defendida por Geraldo Perlino Júnior \\ e aprovada pela comissão julgadora.
}

São Paulo, 26 de agosto de 2005.

Banca examinadora:

- Profa. Dra. Mary Lilian Lourenço IME-USP

- Prof. Dr. Humberto Daniel Carrión Villarroel IME-USP

- Prof. Dr. Ary Orozimbo Chiacchio UNICAMP 
À minha esposa Márcia, aos meus pais Geraldo e Elza e ao meu irmão Wagner, dedico esta dissertação. 


\section{Agradecimentos}

À minha professora e orientadora, Mary Lilian Lourenço, pela sugestão do tema e orientação. Eu aprendi muito com seu estilo de lecionar matemática.

Aos meus pais, Geraldo e Elza, pelo amor deles, pela grande dedicação à minha educação, a eles devoto toda minha admiração, apreço e respeito.

Ao meu irmão, Wagner, por todo o carinho, companheirismo e pelo suporte ao longo da minha formação escolar e da minha vida.

À minha esposa, Márcia, por todos os momentos de alegria, de realizações, de superação nos momentos de dificuldades, de compreensão, de sacrifícios e pelo apoio incondicional em tudo sem o qual esse trabalho não poderia ser realizado.

Aos amigos Fernanda Estevam, Neuza Sanches, Pedro Levit Kauffmann e David Armando Zavaleta Villanueva, que me ajudaram na dissertação de diversas formas. 


\section{Resumo}

O objetivo deste trabalho é estudar a existência de espaços vetoriais gerados por funções patológicas definidas em $\mathbb{R}$, a saber:

- Funções $f: \mathbb{R} \longrightarrow \mathbb{R}$ diferenciáveis em todo domínio mas não monótonas em nenhum intervalo do domínio.

- Funções $f: \mathbb{R} \longrightarrow \mathbb{R}$ ditas fortemente Darboux, ou seja, para qualquer intervalo aberto $I \subset \mathbb{R}, f(I)=\mathbb{R}$.

Além destas funções, estudaremos alguns exemplos de polinômios homogêneos reais definidos em $\mathbb{R}^{m}$ e de polinômios 2-homogêneos definidos em espaços de Banach reais $E$ de dimensão infinita cujos conjuntos de todas as raízes contenham espaços vetoriais. Os resultados estudados aqui estão descritos em [3] e [4].

\section{Abstract}

The main purpose of this work is to study the existence of infinite dimensional vector spaces generated by sets of two pathological functions defined on $\mathbb{R}$, as follow:

- Everywhere differentiable nowhere monotone functions $f: \mathbb{R} \longrightarrow \mathbb{R}$.

- Strongly Darboux functions $f: \mathbb{R} \longrightarrow \mathbb{R}$, i.e., for any open interval $I \subset \mathbb{R}, f(I)=\mathbb{R}$.

Besides, we study some examples of real homogeneous polynomials defined on $\mathbb{R}^{m}$ and 2-homogeneous polynomials defined on infinite dimensional real Banach spaces $E$ whose sets of all zeros contain vector spaces. All the results presented in this work are described in [3] and [4]. 


\section{Índice}

Introdução

1 Conjunto das Funções Diferenciáveis Definidas em $\mathbb{R}$ e Não Monótonas $\begin{array}{ll}\text { em Nenhum Intervalo } & 7\end{array}$

1.1 Definições e Resultados Preliminares . . . . . . . . . . . . . . . . . . . . . 8

1.2 Funções Fat . . . . . . . . . . . . . . . . . . . . . . . . . . . . . . . . . 10

1.3 Existência de Funções DNM(R) . . . . . . . . . . . . . . . . . . . . . . . 18

2 Conjunto das Funções Fortemente Darboux 25

2.1 Funções de Darboux . . . . . . . . . . . . . . . . . . . . . 25

2.2 O Conjunto de Cantor e a Função de Cantor . . . . . . . . . . . . . . . . 29

2.3 Função Fortemente Darboux . . . . . . . . . . . . . . . . . . . . . 33

3 Conjunto das Raízes de Polinômios Homogêneos Reais 41

3.1 Aplicações Multilineares . . . . . . . . . . . . . . . . . . . . . 42

3.2 Polinômios Homogêneos . . . . . . . . . . . . . . . . . . . . . . 47

3.3 Polinômios Homogêneos Definidos em $\mathbb{R}^{m} \ldots \ldots \ldots$. . . . . . . 51 
3.4 Polinômios 2-Homogêneos Definidos em Espaços de Banach de Dimensão Infinita . . . . . . . . . . . . . . . . . . . . . . . . . 59 


\section{Introdução}

"O século $X I X$, que se orgulha da invenção do vapor e da evolução, poderia derivar um título mais legítimo à fama da descoberta da matemática pura."

Bertrand Russell, International Monthly, 1901.

O século $X I X$, conhecido como a Idade do Rigor, foi o marco inicial da fundamentação dos conceitos matemáticos e da descoberta de novas estruturas matemáticas como, por exemplo, o surgimento de uma nova álgebra por Boole e de uma geometria não euclidiana por Lobachevsky.

Nesse contexto, a análise também sofreu profundas e decisivas transformações; entre elas, podemos citar a definição mais precisa de números reais dada por Dedekind e as idéias apresentadas por Weierstrass e Méray de desvincular o cálculo da geometria e baseá-lo somente no conceito de número. A linguagem precisa e lógica tomou lugar dos artifícios heurísticos e conceitos intuitivos; como exemplo desse fato, podemos citar a definição dada por Heine, influenciado por Weierstrass, de limite de uma função por "épsilon e delta" como usamos atualmente. De modo mais incisivo, o próprio conceito de "função" também sofreu modificações desde a primeira metade do século $X I X$; as funções deixaram de ser entes matemáticos puramente atrelados à geometria para tomarem um caráter arbitrário na correspôndencia entre conjuntos se aproximando da definição que conhecemos hoje em dia; uma das confirmações desse fato é o surgimento das funções patológicas, ou seja, funções que violam, pelo menos, uma propriedade válida quase que universalmente (um dos primeiros exemplos é a função de Dirichlet). Esse breve histórico bem como a citação 
acima foram extraídos de [6]. Esses tipos de funções são um dos tópicos que constitui este trabalho de dissertação.

Mais precisamente, este trabalho, dividido em 3 capítulos, tem por objetivo estudar, além da existência de espaços vetoriais contidos nos conjuntos das raízes de polinômios reais homogêneos, a existência de espaços vetoriais contidos em conjuntos de funções patológicas definidas em $\mathbb{R}$ juntamente com a função identicamente nula, isto é, vamos estudar quando um conjunto formado por funções patológicas é lineável (do inglês lineable) cuja definição é apresentada no capítulo 1. Estes tópicos foram estudados nos textos científicos [2], [3], [4] e [14].

No capítulo 1 , estudamos as funções diferenciáveis em todo domínio $\mathbb{R}$ mas não monótonas em nenhum intervalo bem como apresentamos algumas propriedades relativas às derivadas dessas funções; sobre esse assunto, estudamos o texto [15]. Doravante, estas funções serão chamadas de $D N M(\mathbb{R})$. O capítulo se encerra com um teorema sobre a existência de espaços vetoriais formados por funções $D N M(\mathbb{R})$ juntamente com a função identicamente nula. Este tópico está baseado no texto científico [4].

No capítulo 2, estudamos as funções fortemente Darboux começando por apresentar dois exemplos que aparecem em [13]; o primeiro, como fato histórico, dado por Lebesgue (provavelmente ele foi o primeiro matemático a apresentar um exemplo de tal função) e o segundo, mais elaborado, para nos auxiliar na conclusão do capítulo com um resultado sobre a existência de um espaço vetorial gerado por funções fortemente Darboux. Este tópico está baseado no texto científico [4]; observamos que em [4], estas funções são denominadas everywhere surjective functions; porém, usamos o mesmo termo adotado em [9] (funções fortemente Darboux).

No capítulo 3, que é dedicado inteiramente aos polinômios homogêneos definidos em espaços de Banach reais, destacamos as aplicações multilineares estabelecendo um teorema relativo à caracterização de continuidade dessas aplicações com os polinômios homogêneos associados a elas; esse assunto está tratado em [19], referência que estaremos usando no decorrer deste trabalho. Estudamos, também, alguns exemplos de polinômios homogêneos $P: \mathbb{R}^{m} \longrightarrow \mathbb{R}$ cujos conjuntos das raízes contêm espaços vetoriais; esse tópico está baseado em [2] e [3]; por fim, para espaços de Banach $E$ separáveis, verificamos que existem 
polinômios homogêneos $P: E \longrightarrow \mathbb{R}$ tais que os conjuntos $P^{-1}(0)$ contenham espaços vetoriais de dimensão infinita; esse tópico é tratado em [2]. 


\section{,}




\section{Notação}

Vamos usar neste trabalho as seguintes notações:

$\mathbb{N}_{0}: \quad \mathrm{O}$ conjunto dos números naturais

$\mathbb{N}$ : $\quad$ O conjunto dos números inteiros não negativos

$\mathbb{R}: \quad$ O conjunto dos números reais

$\mathbf{B}_{\mathbf{E}}$ : A bola unitária aberta de um espaço normado $E$ sobre $\mathbb{R}$

$\mathrm{rB}_{\mathrm{E}}$ : A bola aberta de raio $r>0$ de um espaço normado $E$ sobre $\mathbb{R}$

$\overline{\mathbf{B}}_{E}$ : A bola unitária fechada de um espaço normado $E$ sobre $\mathbb{R}$

E*: $\quad$ O dual topológico de um espaço normado $E$ munido da norma usual

$\mathbf{E}^{\mathbf{d}}: \quad \quad$ espaço $\underbrace{E \times E \times \ldots \times E}_{d}$

$\mathbf{L}\left(\mathbf{E}_{1}, \ldots, \mathbf{E}_{\mathbf{d}} ; \mathbf{F}\right): \quad$ O espaço das aplicações d-lineares contínuas de $E_{1} \times E_{2} \times \ldots \times E_{d}$ em $F$ sendo $E_{1}, E_{2}, \ldots, E_{d}$ e $F$ espaços normados

$\mathbf{P}\left({ }^{\mathrm{d}} \mathbf{E} ; \mathbf{F}\right): \quad \quad$ espaço dos polinômios d-homogêneos contínuos de $E$ em $F$

$\delta_{\mathrm{ij}}:$

Símbolo de Krönecker sendo $\delta_{i j}=\left\{\begin{array}{ccc}1, & \text { se } i=j \\ 0, & \text { se } i \neq j\end{array}\right.$

$\lambda(\mathrm{M}): \quad$ A lineabilidade do conjunto $M$

$\mathcal{D N} \mathcal{M}(\mathbb{R}): \quad \quad$ O espaço vetorial das funções diferenciáveis e não monótonas em nenhum intervalo de $\mathbb{R}$

$\mathcal{F}(\mathbb{R} ; \mathbb{R}): \quad \quad$ O conjunto de todas as funções com domínio e contra-domínio em $\mathbb{R}$ $\mathcal{F} \mathcal{D}(\mathbb{R}): \quad \quad$ O espaço vetorial das funções fortemente Darboux

c : $\quad$ A cardinalidade do conjunto $\mathbb{R}$ 



\section{Conjunto das Funções Diferenciáveis Definidas em $\mathbb{R}$ e Não Monótonas em Nenhum Intervalo}

O objetivo deste capítulo é estudar a existência de um espaço vetorial de dimensão infinita contido no conjunto formado pela função nula e por todas as funções $f: \mathbb{R} \longrightarrow \mathbb{R}$ diferenciáveis em todo domínio mas não monótonas em nenhum intervalo. Este tópico está baseado no texto científico [4]. Estas funções são chamadas de funções $\operatorname{DNM}(\mathbb{R})$.

O primeiro exemplo de uma função $\operatorname{DNM}(\mathbb{R})$ foi dada por Köpcke em 1889; tal função pode ser vista em [14], pág. 412-421. Seguiram-se vários artigos a respeito deste assunto culminando com um estudo mais aprofundado dado por Denjoy em 1915. Para maiores detalhes, citamos [8] e suas referências. A função de Köpcke é, no entanto, muito elaborada; outros exemplos apareceram mais recentemente (citamos aqui [4] e [15]) com construções mais simples.

No desenvolvimento do estudo de funções $D N M(\mathbb{R})$, usamos [4] como texto básico que, por sua vez, ampliou o trabalho elaborado por Katznelson e Stromberg [15]. Para este estudo, denotamos por $\mathcal{D N} \mathcal{M}(\mathbb{R})$ o conjunto de todas as funções $\operatorname{DNM}(\mathbb{R})$.

Apresentamos, agora, uma definição que servirá de base para a obtenção dos principais resultados: 
Definição 1.1. Seja $X$ o conjunto de todas as funções definidas em $[a ; b]$ ou $\mathbb{R}$. Dada uma determinada propriedade, dizemos que um subconjunto $M$ de $X$ formado por funções que satisfazem tal propriedade é lineável se $M \cup\{0\}$ contiver um espaço vetorial de dimensão infinita e dizemos que é n-lineável se contiver um espaço vetorial de dimensão $n, n \in \mathbb{N}$. Se existir a máxima cardinalidade do espaço vetorial, denotamos por $\lambda(M) e$ dizemos que esta é a lineabilidade (do inglês lineability) de $M$.

O estudo de um conjunto que tem a propriedade de ser lineável, estamos atribuindo, também, a nomenclatura de lineabilidade. Da teoria dos conjuntos, sabe-se que a existência da cardinalidade associada a qualquer conjunto requer o axioma da escolha e que será considerado válido nesse trabalho; portanto, na definição de lineabilidade acima apresentada, a existência da máxima cardinalidade de um espaço vetorial está ligada ao fato de se poder ou não determiná-la.

Posto isso, baseado no texto científico [4], demonstra-se que o conjunto formado por funções $D N M(\mathbb{R})$ é lineável. Para tal demonstração, apresentamos, a seguir, os conceitos e resultados prévios envolvidos de acordo com o referido texto científico.

\subsection{Definições e Resultados Preliminares}

Para o estudo das funções $D N M(\mathbb{R})$ vamos usar conceitos que, em sua maioria, são relativamente simples mas que apresentamos aqui com o objetivo de esclarecer quais resultados vamos usar ao longo deste capítulo. A construção de funções $D N M(\mathbb{R})$ apresentadas neste capítulo é baseada em séries de funções e, portanto, algumas definições e resultados preliminares sobre esse assunto nos auxiliarão no estudo desses tipos de funções.

Definição 1.2. Uma sequência de funções $\left(f_{n}\right)_{n}, f_{n}: A \subseteq \mathbb{R} \longrightarrow \mathbb{R}$ converge uniformemente em A para uma função $f: A \longrightarrow \mathbb{R}$ se, dado $\epsilon>0, \exists n_{o} \in \mathbb{N}$ tal que $\forall n>n_{o} \Longrightarrow\left|f_{n}(x)-f(x)\right|<\epsilon, \forall x \in A$.

Existe um critério para a convergência uniforme de uma seqüência de funções; antes de apresentá-lo, vamos introduzir a seguinte definição: 
Definição 1.3. Uma sequência de funções $\left(f_{n}\right)_{n}, f_{n}: A \subseteq \mathbb{R} \longrightarrow \mathbb{R}$ é chamada de seqüência de Cauchy se, dado $\epsilon>0, \exists n_{o} \in \mathbb{N}$ tal que para quaisquer $m, n>n_{o} \Longrightarrow \sup _{x \in A}\left|f_{n}(x)-f_{m}(x)\right|<\epsilon$.

Segue, então, o critério mencionado:

Teorema 1.4. Uma sequência de funções $\left(f_{n}\right)_{n}, f_{n}: A \subseteq \mathbb{R} \longrightarrow \mathbb{R}$ converge uniformemente em A para $f$ se, somente se, é uma sequência de Cauchy.

Demonstração : $(\Longrightarrow)$ Seja $\left(f_{n}\right)_{n}$ uma seqüência de funções, $f_{n}: A \subseteq \mathbb{R} \longrightarrow \mathbb{R}$, que converge uniformemente em $A$ para $f$. Dado $\epsilon>0$, encontramos $n_{o} \in \mathbb{N}$ tal que $n>n_{o} \Longrightarrow \sup _{x \in A}\left|f_{n}(x)-f(x)\right|<\frac{\epsilon}{2}$. Logo, para todos $m, n>n_{0}, \sup _{x \in A}\left|f_{m}(x)-f_{n}(x)\right| \leq$ $\sup _{x \in A}\left|f_{m}(x)-f(x)\right|+\sup _{x \in A}\left|f(x)-f_{n}(x)\right|<\frac{\epsilon}{2}+\frac{\epsilon}{2}=\epsilon$. Conseqüentemente, $\left(f_{n}\right)_{n}$ é uma sequência de Cauchy.

$(\Longleftarrow)$ Seja $\left(f_{n}\right)_{n}$ de Cauchy. Então, para cada $x \in A,\left(f_{n}(x)\right)_{n}$ é uma seqüência de Cauchy em $\mathbb{R}$. Logo, $\left(f_{n}(x)\right)_{n}$ converge para, digamos, $f(x) \in \mathbb{R}$, isto é, $\lim _{n \rightarrow \infty} f_{n}(x)=f(x), \forall x \in A$. Para mostrar que converge uniformemente em $A$, seja dado $\epsilon>0$; então, $\exists n_{o} \in \mathbb{N}$ tal que $m, n>n_{o} \Longrightarrow \sup _{x \in A}\left|f_{m}(x)-f_{n}(x)\right|<\epsilon$. Fazendo $m \longrightarrow \infty$, obtemos $\sup _{x \in A}\left|f(x)-f_{n}(x)\right|<\epsilon, \forall n>n_{o}$. Logo, $f_{n} \longrightarrow f$ uniformemente em $A$.

O resultado seguinte nos traz um critério de convergência para alguns tipos de série de funções e que será usado posteriormente no teorema 1.16.

Teorema 1.5. (Teste - M de Weierstrass) : Seja $\left(a_{n}\right)_{n}$ uma seqüência de números reais não negativos tal que $\sum_{n=1}^{\infty} a_{n}$ convirja. Seja $\left(f_{n}\right)_{n}, f_{n}: A \subseteq \mathbb{R} \longrightarrow \mathbb{R}$, uma seqüência de funções tal que $\left|f_{n}(x)\right| \leq a_{n}, \forall n \in \mathbb{N}, \forall x \in A$. Então $\sum_{n=1}^{\infty}\left|f_{n}\right| e \sum_{n=1}^{\infty} f_{n}$ são uniformemente convergentes em $A$.

Demonstração : Para a seqüiência $\left(f_{n}\right)_{n}$ dada, seja $\left(\sum_{j=1}^{n} f_{j}\right)_{n}$ uma seqüência de 
funções definidas em $A$. Dado $\epsilon>0$, seja $n_{o} \in \mathbb{N}$ tal que $\sum_{j=n_{0}}^{\infty} a_{j}<\epsilon$. Então, $\forall m, n \in \mathbb{N}$ tais que $m>n>n_{o}$, temos $\left|\sum_{j=1}^{m} f_{j}(x)-\sum_{j=1}^{n} f_{j}(x)\right|=\left|f_{n+1}(x)+\cdots+f_{m}(x)\right| \leq$ $\leq\left|f_{n+1}(x)\right|+\cdots+\left|f_{m}(x)\right| \leq \sum_{j=n+1}^{\infty}\left|f_{j}(x)\right| \leq \sum_{j=n+1}^{\infty} a_{j}<\epsilon, \forall x \in A$. Logo, $\left(\sum_{j=1}^{n}\left|f_{j}\right|\right)_{n} \mathrm{e}$ $\left(\sum_{j=1}^{n} f_{j}\right)_{n}$ são seqüências de Cauchy e, pelo teorema 1.4 , segue o resultado.

\subsection{Funções Fat}

A principal ferramenta para provarmos que o conjunto $\mathcal{D N} \mathcal{M}(\mathbb{R})$ das funções diferenciáveis em $\mathbb{R}$ mas não monótonas em nenhum intervalo de $\mathbb{R}$ é lineável é um teorema relativo à diferenciação de séries uniformemente convergentes. A proposta desta seção é apresentar este teorema; para isso, vamos usar funções com determinadas propriedades que definiremos a seguir:

Definição 1.6. Seja $f: \mathbb{R} \longrightarrow \mathbb{R}$ uma função integrável em todo intervalo $[a ; b] \subset \mathbb{R}$. Se existe $H \in(0 ; \infty)$ tal que, para cada $a<b$,

$$
\left(\frac{1}{b-a}\right)\left|\int_{a}^{b} f(t) d t\right| \leq H \cdot \min \{|f(a)|,|f(b)|\}
$$

dizemos que $f$ é fat.

Definições 1.7. Seja $f$ uma função fat para algum $H>0$ dado em (1.1). O número $H_{f}=\inf (H)$ é chamado de fatness da função $f$. Uma família $\mathcal{F}$ de funções fat é chamada de uniformemente fat se

$$
H_{\mathcal{F}}=\sup _{f \in \mathcal{F}}\left(H_{f}\right)<\infty
$$

Grosso modo, se uma função é fat, então o seu valor médio sobre qualquer intervalo não pode ser " muito maior " quando comparado aos seus valores nos extremos deste intervalo. 
Uma outra propriedade especial relativa às funções é apresentada a seguir:

Definição 1.8. Uma função $\varphi: \mathbb{R} \longrightarrow \mathbb{R}$ par, positiva e contínua tal que $\varphi(|x|) \downarrow 0$ quando $x \longrightarrow \infty$ é chamada de função escalada.

Observação: $\varphi(|x|) \downarrow 0$ quando $x \longrightarrow \infty$ é equivalente a $\varphi$ ser crescente em $(-\infty ; 0]$ e decrescente em $[0 ; \infty)$ e $\lim _{|x| \longrightarrow \infty} \varphi(x)=0$.

Exemplos 1.9. Como exemplos de funções escaladas podemos citar:

a) $f(x)=e^{-|x|}$

b) $f(x)=(1+|x|)^{-\alpha}, \forall \alpha \in(0 ; \infty)$

O próximo resultado nos dá uma condição suficiente para o fatness:

Proposição 1.10. Seja $\varphi$ uma função escalada; se existe $K>0$ tal que para todo $b>0$,

$$
\frac{1}{b} \int_{0}^{b} \varphi(t) d t \leq K \varphi(b),
$$

então $\varphi$ é fat e $H_{\varphi} \leq 2 K$, sendo $H_{\varphi}$ o fatness da função $\varphi$. Para $0<a<b$, da desigualdade (1.1) podemos tomar $H=K$.

Demonstração : Seja $[a ; b] \subset \mathbb{R}, a<b$, um intervalo qualquer de $\mathbb{R}$. Como $\varphi$ é uma função par, podemos supor, sem perda de generalidade, que $|a|<b$. Há, então, 2 casos a considerar:

$$
\begin{aligned}
& \text { i) }-b<a \leq 0 \text { : } \\
& \qquad\left(\frac{1}{b-a}\right) \int_{a}^{b} \varphi(t) d t=\left(\frac{1}{b-a}\right)\left(\int_{a}^{0} \varphi(t) d t+\int_{0}^{b} \varphi(t) d t\right)= \\
& \left(\frac{1}{b-a}\right)\left(\int_{0}^{-a} \varphi(t) d t+\int_{0}^{b} \varphi(t) d t\right) \leq \frac{2}{(b-a)} \int_{0}^{b} \varphi(t) d t \leq \frac{2}{(b-a)} b \cdot K \cdot \varphi(b) \leq 2 K \varphi(b) \\
& \text { ii) } 0 \leq a<b \text { : }
\end{aligned}
$$

Fazendo a substituição linear $t=t(x)=a+\left(\frac{b-a}{b}\right) x$, temos: 
$t(0)=a, t(b)=b$ e $t(x) \geq x, \forall x \in[0 ; b]$ pois $t(x)-x=a\left(\frac{b-x}{b}\right) \geq 0$.

Como $\varphi$ é decrescente em $[0 ; \infty), \varphi(t(x)) \leq \varphi(x)$. Portanto:

$$
\left(\frac{1}{b-a}\right) \int_{a}^{b} \varphi(t) d t=\left(\frac{1}{b}\right) \int_{0}^{b} \varphi(t(x)) d x \leq \frac{1}{b} \int_{0}^{b} \varphi(x) d x \leq K \cdot \varphi(b)
$$

Logo, $\varphi$ é fat e $H_{\varphi} \leq 2 K$.

Exemplo 1.11. Toda função escalada da forma $\varphi(t)=(1+|t|)^{-\alpha}, 0<\alpha<1$ é fat.

De fato, seja $b>0$; então:

$$
\begin{gathered}
{\left[\frac{1}{b \varphi(b)}\right] \int_{0}^{b} \frac{d t}{(1+t)^{\alpha}}=\left.\frac{1}{b \varphi(b)} \cdot \frac{(1+t)^{(1-\alpha)}}{(1-\alpha)}\right|_{0} ^{b}=\frac{(b+1)^{\alpha}}{b(1-\alpha)}\left[(b+1)^{(1-\alpha)}-1\right]=} \\
=\frac{b+1-(b+1)^{\alpha}}{b(1-\alpha)}=\frac{1}{1-\alpha}-\frac{(b+1)^{\alpha}-1}{b(1-\alpha)}<\frac{1}{1-\alpha}
\end{gathered}
$$

Então, $\quad H_{\varphi} \leq \frac{2}{1-\alpha}$.

Através de uma função escalada, podemos construir um conjunto de funções a saber: Definição 1.12. Para uma função escalada $\varphi$, seja $L(\varphi)$ o conjunto das funções da forma $\sum_{j=1}^{n} c_{j} \varphi\left(\lambda_{j}\left(x-\alpha_{j}\right)\right)$ com $n \in \mathbb{N}, c_{j}, \lambda_{j}$ reais positivos e $\alpha_{j} \in \mathbb{R}, \forall j=1,2, \cdots, n$. As funções em $L(\varphi)$ são chamadas de $\varphi$-funções.

Exemplo 1.13. Considere $\varphi(t)=\frac{1}{\sqrt{1+|t|}}, c_{j}=\lambda_{j}=1,1 \leq j \leq 3$ e $\alpha_{1}=-1, \alpha_{2}=0 e$ $\alpha_{3}=1$; desta forma, um dos elementos de $L(\varphi)$ é dado pela função $\Psi$ expressa da seguinte maneira:

$$
\Psi(t)=\frac{1}{\sqrt{1+|t+1|}}+\frac{1}{\sqrt{1+|t|}}+\frac{1}{\sqrt{1+|t-1|}}
$$




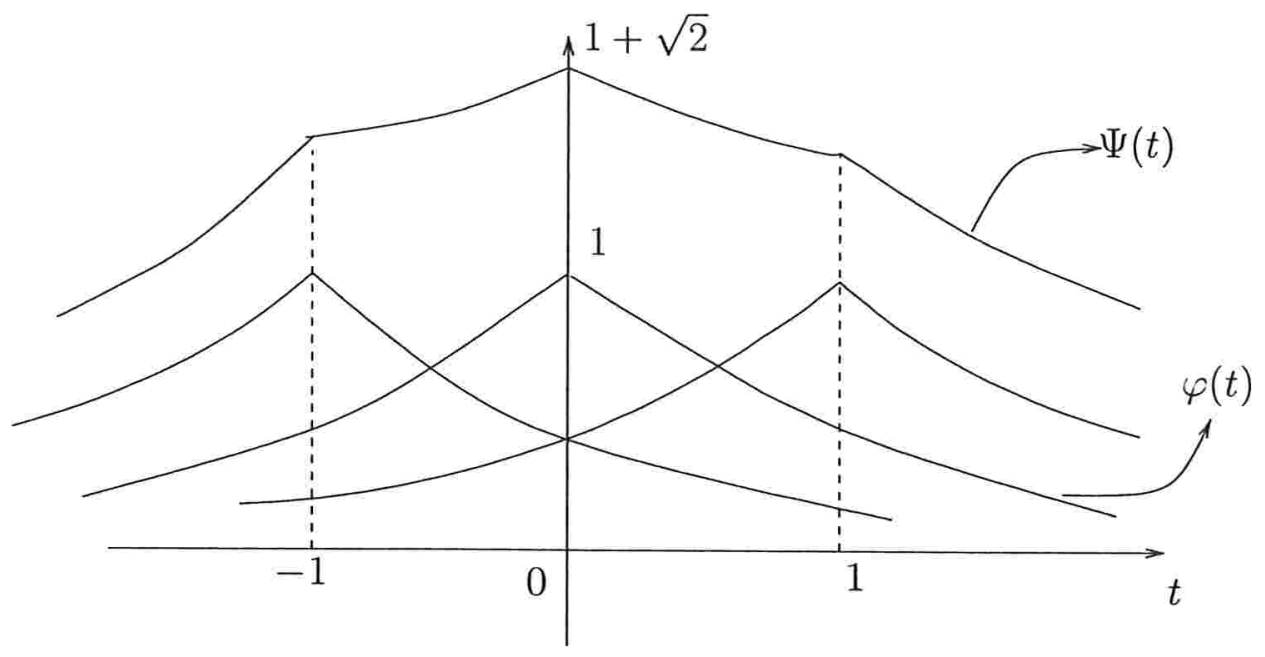

Figura 1.1: Esboço dos gráficos das funções $\varphi$ e $\Psi$

Para que $L(\varphi)$ seja uniformemente fat, basta impor apenas uma condição sobre $\varphi$ :

Proposição 1.14. Se uma função escalada $\varphi$ é fat, então a família de funções $L(\varphi)$ é uniformemente fat. Além disso, $H_{\varphi}=H_{L(\varphi)}$.

Demonstração : Considere $\Psi(x)=\sum_{j=1}^{n} c_{j} \varphi\left(\lambda_{j}\left(x-\alpha_{j}\right)\right), \Psi \in L(\varphi)$ e $[a ; b] \subset \mathbb{R}$ arbitrários. Considere $A:=\frac{1}{(b-a)} \int_{a}^{b} \Psi(x) d x$. Então:

$$
A=\frac{1}{(b-a)} \int_{a}^{b} \sum_{j=1}^{n} c_{j} \varphi\left(\lambda_{j}\left(x-\alpha_{j}\right)\right) d x=\sum_{j=1}^{n} \frac{c_{j}}{(b-a)} \int_{a}^{b} \varphi\left(\lambda_{j}\left(x-\alpha_{j}\right)\right) d x .
$$

Vamos estabelecer as seguintes mudanças de variáveis $y_{j}=y_{j}(x)=\lambda_{j}\left(x-\alpha_{j}\right)$,

$\forall j=1,2, \cdots, n$. Com isso, $d y_{j}=\lambda_{j} d x, \forall j=1,2, \cdots, n$ e é claro que $\frac{y_{j}(b)-y_{j}(a)}{\lambda_{j}(b-a)}=1$,

$\forall j=1,2, \cdots, n . \operatorname{Assim} A=\sum_{j=1}^{n} \frac{c_{j}}{\lambda_{j}(b-a)} \int_{y_{j}(a)}^{y_{j}(b)} \varphi\left(y_{j}\right) d y_{j}$.

Como a função $\varphi$ é fat, temos: 
$A \leq \sum_{j=1}^{n} \frac{c_{j}\left[y_{j}(b)-y_{j}(a)\right]}{\lambda_{j}(b-a)} \cdot H_{\varphi} \cdot \min \left\{\varphi\left(y_{j}(b)\right), \varphi\left(y_{j}(a)\right)\right\}=H_{\varphi} \sum_{j=1}^{n} c_{j} \cdot \min \left\{\varphi\left(y_{j}(b)\right), \varphi\left(y_{j}(a)\right)\right\}$

Mas:

$$
\Psi(a)=\sum_{j=1}^{n} c_{j} \varphi\left(y_{j}(a)\right) \geq \sum_{j=1}^{n} c_{j} \min \left\{\varphi\left(y_{j}(b)\right), \varphi\left(y_{j}(a)\right)\right\}
$$

e

$$
\Psi(b)=\sum_{j=1}^{n} c_{j} \varphi\left(y_{j}(b)\right) \geq \sum_{j=1}^{n} c_{j} \min \left\{\varphi\left(y_{j}(b)\right), \varphi\left(y_{j}(a)\right)\right\}
$$

De (1.2), (1.3) e (1.4) temos que $A=\frac{1}{(b-a)} \int_{a}^{b} \Psi(x) d x \leq H_{\varphi} \min \{\Psi(a) ; \Psi(b)\}$. Assim $\Psi$ é fat.

Sendo $\Psi$ arbitrária, temos que $H_{\Psi} \leq H_{\varphi}, \forall \Psi \in L(\varphi)$, ou seja, $H_{L(\varphi)}=\sup _{\Psi \in L(\varphi)} H_{\Psi} \leq H_{\varphi}$. Assim, $L(\varphi)$ é uniformemente fat e como $\varphi \in L(\varphi)$, segue que $H_{\varphi}=H_{L(\varphi)}$.

Para uma função escalada $\varphi$ dada, podemos exibir $\varphi$-funções com determinadas propriedades como mostra a proposição seguinte e que serão úteis na próxima seção em que se mostra a existência de funções $D N M(\mathbb{R})$.

Proposição 1.15. (Flexibilidade de $\mathrm{L}(\varphi)$ ): Sejam $\varphi$ uma função escalada arbitrária, $n \in \mathbb{N}, n$ números reais distintos $\left\{\alpha_{j}\right\}_{j=1}^{n}$ e $n$ intervalos abertos $\left\{I_{j}=\left(y_{j}, \tilde{y}_{j}\right)\right\}_{j=1}^{n}$ com $0<y_{j}<\tilde{y}_{j}, \forall j=1,2, \cdots, n$. Então existe uma função $\Psi \in L(\varphi)$ tal que:
i) $\Psi\left(\alpha_{j}\right) \in I_{j}, \forall j=1,2, \cdots, n$
ii) $\Psi(x)<\max _{1 \leq j \leq n}\left\{\tilde{y}_{j}\right\}, \forall x \in \mathbb{R}$

Demonstração : i) Considere $J_{j}$ intervalos contendo $\alpha_{j}, 1 \leq j \leq n$ dois a dois disjuntos. Considere, também, $\epsilon>0$ tal que $\epsilon<\min _{1 \leq j \leq n}\left\{\left|\tilde{y}_{j}-y_{j}\right|\right\}$. Definimos as funçōes 
$\Psi_{j}(x)=c_{j} \varphi\left(\lambda_{j}\left(x-\alpha_{j}\right)\right), \forall j=1,2, \cdots, n$ tais que $c_{j}=\frac{y_{j}+\epsilon / 2}{\varphi(0)}$ e com os $\lambda_{j}$ suficientemente grandes tais que $\Psi_{j}(x) \leq \frac{\epsilon}{2 n}, \quad \forall x \notin J_{j}$. Por fim, considere $\Psi(x)=\sum_{j=1}^{n} \Psi_{j}(x)$, $\forall x \in \mathbb{R}$.

Então, $\Psi_{j}\left(\alpha_{j}\right)=c_{j} \varphi(0)=y_{j}+\frac{\epsilon}{2}$. Como $\Psi_{i}\left(\alpha_{j}\right)>0$, segue que $\Psi\left(\alpha_{j}\right)>y_{j}$.

Por outro lado,

$$
\begin{gathered}
\Psi\left(\alpha_{j}\right)=\Psi_{j}\left(\alpha_{j}\right)+\sum_{\substack{i=1 \\
i \neq j}}^{n} \Psi_{i}\left(\alpha_{j}\right)=y_{j}+\frac{\epsilon}{2}+\sum_{\substack{i=1 \\
i \neq j}}^{n} \Psi_{i}\left(\alpha_{j}\right) \leq y_{j}+\frac{\epsilon}{2}+\frac{\epsilon}{2 n}(n-1)= \\
=y_{j}+\epsilon-\frac{\epsilon}{2 n}<y_{j}+\epsilon<y_{j}+\tilde{y}_{j}-y_{j}=\tilde{y}_{j} .
\end{gathered}
$$

Assim, $\Psi\left(\alpha_{j}\right) \in I_{j}$.

ii) Primeiramente, vamos considerar $x \in J_{i}$ para algum $i, 1 \leq i \leq n$. Lembrando-se que $\varphi(x) \leq \varphi(0), \forall x \in \mathbb{R}$, temos:

$$
\begin{aligned}
\Psi(x)=\Psi_{i}(x)+ & \sum_{\substack{k=1 \\
k \neq i}}^{n} \Psi_{k}(x)=\frac{y_{i}+\frac{\epsilon}{2}}{\varphi(0)} \cdot \varphi\left(\lambda_{i}\left(x-\alpha_{i}\right)\right)+\sum_{\substack{k=1 \\
k \neq i}}^{n} \Psi_{j}(x) \leq \\
& \leq y_{i}+\frac{\epsilon}{2}+\frac{\epsilon}{2 n}(n-1)<y_{i}+\epsilon<\tilde{y}_{i}
\end{aligned}
$$

Assim, como $x$ foi escolhido arbitrariamente em $J_{i}$, segue que $\Psi(x)<\tilde{y}_{i}$, para todo $x \in J_{i}$. Como $J_{i}$ foi escolhido arbitrariamente, segue $\Psi(x)<\max _{1 \leq i \leq n} \tilde{y}_{i}$, para todo $x \in \bigcup_{i=1}^{n} J_{i}$.

Para $x \notin \bigcup_{i=1}^{n} J_{i}$, temos que $\Psi(x)=\sum_{j=1}^{n} \Psi_{j}(x) \leq \frac{\epsilon}{2 n} \cdot n=\frac{\epsilon}{2}<\epsilon$. Como $\epsilon<\tilde{y}_{j}$ para todo $1 \leq j \leq n$, conseqüentemente $\Psi(x)<\max _{1 \leq i \leq n}\left\{\tilde{y}_{i}\right\}$, para todo $x \in \mathbb{R}$.

Para demonstrar que o conjunto $\mathcal{D} \mathcal{N} \mathcal{M}(\mathbb{R})$ é lineável, vamos apresentar o teorema a seguir referente à diferenciação de séries uniformemente convergentes, porém envolvendo uma família de funções fat. Este resultado é análogo ao teorema clássico de diferenciação de séries e que pode ser encontrado, por exemplo, em [18], pg. 304. 
Teorema 1.16. Seja $\sum_{n=1}^{\infty} \Phi_{n}(x)$ uma série de funções sobre $\mathbb{R}$ de classe $C^{1}$ tal que, para algum $x_{o} \in \mathbb{R}, \sum_{n=1}^{\infty} \Phi_{n}\left(x_{o}\right)$ convirja. Para cada $n$, seja $\Psi_{n}=\Phi_{n}^{\prime}$. Se $\left\{\Psi_{n}: n \in \mathbb{N}\right\}$ é uma seqüência de funções positivas uniformemente fat com $\sum_{n=1}^{\infty} \Psi_{n}(a)=s<\infty$, para algum $a \in \mathbb{R}$, então:

i) $\sum_{n=1}^{\infty} \Phi_{n}(x)=F(x)$ é uniformemente convergente em cada intervalo limitado de $\mathbb{R}$.

ii) $F^{\prime}(a)=s$.

Em particular, se $\sum_{n=1}^{\infty} \Psi_{n}(x)=s(x)<\infty, \forall x \in \mathbb{R}$, então $F^{\prime}(x)=s(x), \forall x \in \mathbb{R}$.

Demonstração : i) Considere $b \geq \max \left\{|a|,\left|x_{o}\right|\right\}$ e seja $H=\sup _{n \in \mathbb{N}} H_{n} \operatorname{com} H_{n}$ sendo o fatness de $\Psi_{n}, \forall n$. Para $|x| \leq b$, segue

$$
\begin{gathered}
\left|\Phi_{n}(x)-\Phi_{n}(0)\right| \leq\left|\int_{0}^{a} \Psi_{n}(t) d t\right|+\left|\int_{a}^{x} \Psi_{n}(t) d t\right| \leq \\
\leq|a| \cdot H \cdot \Psi_{n}(a)+|a-x| \cdot H \cdot \Psi_{n}(a) \leq(2|a|+|x|) \cdot H \cdot \Psi_{n}(a) \leq 3 b \cdot H \cdot \Psi_{n}(a) ;
\end{gathered}
$$

então, pelo teste-M de Weierstrass (ver teorema 1.5), a série $\sum_{n=1}^{\infty}\left[\Phi_{n}(x)-\Phi_{n}(0)\right]$ converge uniformemente em $[-b ; b]$. Como, por hipótese, $\sum_{n=1}^{\infty} \Phi_{n}\left(x_{o}\right)$ converge, podemos escrever $\sum_{n=1}^{\infty} \Phi_{n}(0)=\sum_{n=1}^{\infty} \Phi_{n}\left(x_{0}\right)-\sum_{n=1}^{\infty}\left[\Phi_{n}\left(x_{0}\right)-\Phi_{n}(0)\right] . \quad$ Logo, $\sum_{n=1}^{\infty} \Phi_{n}(0)$ converge e, conseqüentemente, $\sum_{n=1}^{\infty} \Phi_{n}(x)$ é uniformemente convergente em $[-b ; b]$ para alguma função $F$. Assim, a afirmação $i$ ) está provada.

ii) Dado $\epsilon>0$ arbitrário, considere $N \in \mathbb{N}$ tal que $\sum_{n=N+1}^{\infty} \Psi_{n}(a)<\frac{\epsilon}{2(H+1)}$.

Pela continuidade das $\Psi_{n}, \forall n \in \mathbb{N}$, existe um $\delta>0$ tal que, $\forall h \in \mathbb{R}$ com $|h|<\delta$ e 
$n \leq N$, tenhamos:

$$
\left|\Psi_{n}(a+h)-\Psi_{n}(a)\right|<\frac{\epsilon}{2 N}
$$

Considerando, sem perda de generalidade, que $h>0$ (para $h<0$ a demonstração é análoga), obtemos:

$$
\left|\frac{1}{h} \int_{a}^{a+h} \Psi_{n}(t) d t-\Psi_{n}(a)\right| \leq \frac{1}{h} \int_{a}^{a+h}\left|\Psi_{n}(t)-\Psi_{n}(a)\right| d t
$$

Pela desigualdade (1.5), temos:

$$
\left|\frac{1}{h} \int_{a}^{a+h} \Psi_{n}(t) d t-\Psi_{n}(a)\right| \leq \frac{1}{h} \cdot \frac{\epsilon}{2 N} \cdot h=\frac{\epsilon}{2 N}
$$

Como, por hipótese, $\Phi_{n}^{\prime} \equiv \Psi_{n}: n \in \mathbb{N}$, segue que:

$$
\begin{gathered}
\left|\frac{F(a+h)-F(a)}{h}-s\right|= \\
=\left|\sum_{n=1}^{\infty} \frac{\left[\Phi_{n}(a+h)-\Phi_{n}(a)\right]}{h}-\sum_{n=1}^{\infty} \Psi_{n}(a)\right|=\left|\sum_{n=1}^{\infty} \frac{1}{h} \int_{a}^{a+h} \Psi_{n}(t) d t-\sum_{n=1}^{\infty} \Psi_{n}(a)\right|= \\
=\left|\sum_{n=1}^{\infty}\left[\frac{1}{h} \int_{a}^{a+h} \Psi_{n}(t) d t-\Psi_{n}(a)\right]\right| \leq \\
\leq\left|\sum_{n=1}^{N}\left[\frac{1}{h} \int_{a}^{a+h} \Psi_{n}(t) d t-\Psi_{n}(a)\right]\right|+\left|\sum_{n=N+1}^{\infty}\left[\frac{1}{h} \int_{a}^{a+h} \Psi_{n}(t) d t-\Psi_{n}(a)\right]\right| \leq \\
\leq \sum_{n=1}^{N}\left|\frac{1}{h} \int_{a}^{a+h} \Psi_{n}(t) d t-\Psi_{n}(a)\right|+\sum_{n=N+1}^{\infty}\left[\frac{1}{h} \int_{a}^{a+h} \Psi_{n}(t) d t\right]+\left|\sum_{n=N+1}^{\infty} \Psi_{n}(a)\right|
\end{gathered}
$$

De (1.6) e do fato de $\left\{\Psi_{n}: n \in \mathbb{N}\right\}$ é uniformemente fat, temos:

$$
\begin{gathered}
\left|\frac{F(a+h)-F(a)}{h}-s\right| \leq \sum_{n=1}^{N} \frac{\epsilon}{2 N}+\sum_{n=N+1}^{\infty} H \cdot \Psi_{n}(a)+\frac{\epsilon}{2(H+1)}< \\
<\frac{\epsilon}{2 N} \cdot N+\frac{H \epsilon}{2(H+1)}+\frac{\epsilon}{2(H+1)}=\frac{\epsilon}{2}+\frac{\epsilon}{2}=\epsilon
\end{gathered}
$$




\subsection{Existência de Funções DNM(R)}

Nesta seção, vamos apresentar um resultado sobre a existência de funções $D N M(\mathbb{R})$ usando sequiência uniformemente fat de $\varphi$-funções. O objetivo é mostrar que o conjunto $\mathcal{D N} \mathcal{M}(\mathbb{R})$ é lineável.

Teorema 1.17. Seja $\left(y_{n}\right)_{n}$ uma seqüência estritamente crescente de números reais convergindo para 1 tal que $y_{0}=0$. Seja $S_{o}=\left\{\alpha_{j}^{(o)}\right\}_{j=1}^{\infty}$ uma seqüência de números reais dois a dois distintos e, para cada $i \in \mathbb{N}$, seja $S_{i}=\left\{\alpha_{j}^{(i)}\right\}_{j=1}^{m_{i}}$ um conjunto finito de números reais dois a dois distintos. Além disso, suponhamos que os conjuntos $\left\{S_{i}\right\}_{i=0}^{\infty}$ sejam dois a dois disjuntos. Então, existe uma função $F$ diferenciável em $\mathbb{R}$ tal que:

i) $0<F^{\prime}(x) \leq 1, \quad$ para todo $\quad x \in \mathbb{R}$.

ii) $F^{\prime}\left(\alpha_{j}^{(o)}\right)=1, \quad$ para todo $j \in \mathbb{N}$

iii) $\quad F^{\prime}\left(\alpha_{j}^{(i)}\right)=y_{i}, \quad$ para todo $j=1,2, \cdots, m_{i}$ e $i=1,2, \cdots$

Demonstração : Para cada $i \in \mathbb{N}$ e cada intervalo $I_{i}=\left(y_{i-1} ; y_{i}\right)$, consideremos a seqüência $\left(y_{i, k}\right)_{k}$ estritamente crescente tal que $y_{i, k} \in I_{i}, \forall k \in \mathbb{N}_{0}$ e $\lim _{k \rightarrow \infty} y_{i, k}=y_{i}$. Seja $\varphi$ uma função escalada com a mesma propriedade apresentada na proposição 1.10. Tomando-se o intervalo $\left(y_{1 ; 0} ; y_{1 ; 1}\right)$ e o conjunto $S_{1} \cup\left\{\alpha_{1}^{(0)}\right\}$, temos que, pela proposição 1.15 , existe uma função $f_{1}=\Psi_{1} \in L(\varphi)$ tal que:

I.1) $f_{1}\left(\alpha_{j}^{(1)}\right) \in\left(y_{1,0} ; y_{1,1}\right)$, para $j=1,2 \cdots m_{1}$.

I.2) $f_{1}\left(\alpha_{1}^{(0)}\right) \in\left(y_{1,0} ; y_{1,1}\right)$.

I.3) $f_{1}(t)<y_{1.1}, \quad \forall t \in \mathbb{R}$.

Tomando-se, agora, os intervalos $\left(y_{1 ; 1}-\Psi_{1}\left(\alpha_{j}^{(1)}\right) ; y_{1 ; 2}-\Psi_{1}\left(\alpha_{j}^{(1)}\right)\right), j=1,2, \cdots, m_{1}$, $\left(y_{2 ; 1}-\Psi_{1}\left(\alpha_{j}^{(2)}\right) ; y_{2 ; 2}-\Psi_{1}\left(\alpha_{j}^{(2)}\right)\right), j=1,2, \cdots, m_{2}$ e $\left(y_{2 ; 1}-\Psi_{1}\left(\alpha_{j}^{(0)}\right) ; y_{2 ; 2}-\Psi_{1}\left(\alpha_{j}^{(0)}\right)\right)$, $j=1,2$ e o conjunto $S_{1} \cup S_{2} \cup\left\{\alpha_{1}^{(0)} ; \alpha_{2}^{(0)}\right\}$. novamente pela proposição 1.15 , existe uma função $\Psi_{2} \in L(\varphi)$ tal que: 

a) $\Psi_{2}\left(\alpha_{j}^{(1)}\right) \in\left(y_{1 ; 1}-\Psi_{1}\left(\alpha_{j}^{(1)}\right) ; y_{1 ; 2}-\Psi_{1}\left(\alpha_{j}^{(1)}\right)\right)$ para $j=1,2, \cdots, m_{1}$
b) $\Psi_{2}\left(\alpha_{j}^{(2)}\right) \in\left(y_{2 ; 1}-\Psi_{1}\left(\alpha_{j}^{(2)}\right) ; y_{2 ; 2}-\Psi_{1}\left(\alpha_{j}^{(2)}\right)\right) \operatorname{para} j=1,2, \cdots, m_{2}$
c) $\quad \Psi_{2}\left(\alpha_{j}^{(0)}\right) \in\left(y_{2 ; 1}-\Psi_{1}\left(\alpha_{j}^{(0)}\right) ; y_{2 ; 2}-\Psi_{1}\left(\alpha_{j}^{(0)}\right)\right)$ para $j=1,2$
d) $\Psi_{2}(t)<\max _{\substack{1 \leq j \leq m_{1} \\ 1 \leq 1 \leq m_{2} \\ 1 \leq s \leq 2}}\left\{\left(y_{1 ; 2}-\Psi_{1}\left(\alpha_{j}^{(1)}\right)\right),\left(y_{2 ; 2}-\Psi_{1}\left(\alpha_{l}^{(2)}\right)\right),\left(y_{2 ; 2}-\Psi_{1}\left(\alpha_{s}^{(0)}\right)\right)\right\}, \forall t \in \mathbb{R}$.

Definimos, então, a função $f_{2}=\Psi_{1}+\Psi_{2}$. Com isso, temos:

II.1) $f_{2}\left(\alpha_{j}^{(1)}\right) \in\left(y_{1,1} ; y_{1,2}\right)$, para $j=1,2 \cdots m_{1}$ $f_{2}\left(\alpha_{j}^{(2)}\right) \in\left(y_{2,1} ; y_{2,2}\right)$, para $j=1,2 \cdots m_{2}$

II.2) $f_{2}\left(\alpha_{j}^{(0)}\right) \in\left(y_{2,1} ; y_{2,2}\right)$ para $j=1,2$

II.3) $\quad f_{2}(t)<y_{2.2}, \forall t \in \mathbb{R}$.

Procedendo de forma indutiva, podemos construir uma seqüência $\left(\Psi_{n}\right)_{n}$ com $\Psi_{n} \in L(\varphi), \forall n \in \mathbb{N}$ e, portanto, obtermos uma seqüência $\left(f_{n}\right)_{n} \operatorname{com} f_{n} \equiv \sum_{k=1}^{n} \Psi_{k}$, $n=1,2, \cdots$ tal que:

$$
\begin{array}{lll}
\text { N.1) } & f_{n}\left(\alpha_{j}^{(1)}\right) \in\left(y_{1, n-1} ; y_{1, n}\right), \text { para } j=1,2 \cdots m_{1} \\
& f_{n}\left(\alpha_{j}^{(2)}\right) \in\left(y_{2, n-1} ; y_{2, n}\right), \text { para } j=1,2 \cdots m_{2} \\
& \vdots \\
& f_{n}\left(\alpha_{j}^{(n)}\right) \in\left(y_{n, n-1} ; y_{n, n}\right), \quad \text { para } j=1,2 \cdots m_{n} \\
N .2) & f_{n}\left(\alpha_{j}^{(0)}\right) \in\left(y_{n, n-1} ; y_{n, n}\right), \quad \text { para } j=1,2 \cdots, n \\
\text { N.3) } & f_{n}(t)<y_{n, n}, \quad \forall t \in \mathbb{R} .
\end{array}
$$

Por construção, $f_{n}(t)<1$ e $\Psi_{n}(t)>0, \forall t \in \mathbb{R}, \forall n \in \mathbb{N}$. Então, $\forall t \in \mathbb{R}$, a série $\sum_{n=1}^{\infty} \Psi_{n}(t)$ converge ( toda seqüência monótona e limitada é convergente) para, digamos, $f(t)$. Como $\Psi_{n}$ é contínua e limitada em $\mathbb{R}$, podemos definir $\Phi_{n}(x)=\int_{0}^{x} \Psi_{n}(t) d t, \forall n \in \mathbb{N}$. Desta forma, temos as seguintes condições: 
- Pela proposição $1.14,\left(\Psi_{n}\right)_{n}$ é uma seqüência uniformemente fat de funções positivas cuja série $\sum_{n=1}^{\infty} \Psi_{n}(t)$ converge para $f(t), \forall t \in \mathbb{R}$.

- Para qualquer $b>0$, considerando $|x| \leq b$, temos $\left|\Phi_{n}(x)\right|=\left|\int_{0}^{x} \Psi_{n}(t) d t\right| \leq$ $|x| \cdot H \cdot \Psi_{n}(b) \leq b \cdot H \cdot \Psi_{n}(b)$; pelo teste-M de Weierstrass, a série $\sum_{n=1}^{\infty} \Phi_{n}(x)$ converge uniformemente em $[-b ; b]$.

- Pelo teorema 1.16, existe uma função $F$ tal que $F(x)=\sum_{n=1}^{\infty} \Phi_{n}(x)$ e $F^{\prime}(x)=f(x)$. Segue, então:

i) $0<F^{\prime}(x)=\lim _{n \rightarrow \infty} f_{n}(x) \leq 1, \quad \forall x \in \mathbb{R}$

ii) $1=\lim _{n \rightarrow \infty} y_{n, n-1} \leq \lim _{n \rightarrow \infty} f_{n}\left(\alpha_{j}^{(0)}\right)=F^{\prime}\left(\alpha_{j}^{(0)}\right) \leq \lim _{n \rightarrow \infty} y_{n, n}=1 \Longrightarrow F^{\prime}\left(\alpha_{j}^{(0)}\right)=1$, $\forall j \in \mathbb{N}$

iii) $\quad y_{i}=\lim _{n \rightarrow \infty} y_{i, n-1} \leq \lim _{n \rightarrow \infty} f_{n}\left(\alpha_{j}^{(i)}\right)=F^{\prime}\left(\alpha_{j}^{(i)}\right) \leq \lim _{n \rightarrow \infty} y_{i, n}=y_{i}, j=1,2, \ldots, m_{i}$ e

$$
i=1,2, \ldots
$$

Logo, $\quad F^{\prime}\left(\alpha_{j}^{(i)}\right)=y_{i}, \forall j=1,2, \cdots, m_{i}$ e $i=1,2, \cdots$

Como conseqüência deste teorema, temos o seguinte resultado:

Teorema 1.18. Sejam $A^{+}, A^{-}, A^{o}$ subconjuntos enumeráveis de $\mathbb{R}$ disjuntos dois a dois. Então, existe uma função $H$ diferenciável em $\mathbb{R}$ tal que $\left|H^{\prime}(x)\right| \leq 1, \forall x \in \mathbb{R}$ e:
i) $H^{\prime}(x)>0$, se $x \in A^{+}$
ii) $\quad H^{\prime}(x)<0$, se $x \in A^{-}$
iii) $\quad H^{\prime}(x)=0$, se $x \in A^{o}$ 
Demonstração : Seja $\left(y_{n}\right)_{n}$ uma seqüência estritamente crescente de números reais convergindo para 1 tal que $y_{0}=0$. Para cada $i \in \mathbb{N}$ e cada intervalo $I_{i}=\left(y_{i-1} ; y_{i}\right)$, consideremos a seqüência $\left(y_{i, k}\right)_{k}$ estritamente crescente tal que $y_{i, k} \in I_{i}, \forall k \in \mathbb{N}_{0}$ e $\lim _{k \rightarrow \infty} y_{i, k}=y_{i}$. Seja $\varphi$ uma função escalada dada como na proposição 1.10. Sejam $A^{0}, A^{+}=\bigcup_{i=1}^{\infty} S_{i}^{+}$e $A^{-}=\bigcup_{i=1}^{\infty} S_{i}^{-}$subconjuntos enumeráveis de $\mathbb{R}$ dois a dois disjuntos, sendo $S_{i}^{+}=\left\{\alpha_{j}^{(i)}\right\}_{j=1}^{m_{i}}$ e $S_{i}^{-}=\left\{\beta_{j}^{(i)}\right\}_{j=1}^{n_{i}}$ conjuntos finitos de números reais para todo $i \in \mathbb{N}$. Pelo teorema 1.17, existem duas funções diferenciáveis $F$ e $G$ tais que:

$$
\begin{array}{rlrlrl}
\text { a) } F^{\prime}(x) & =1, & \forall x \in A^{+} \cup A^{0} & \text { b) } G^{\prime}(x)=1, & \forall x \in A^{-} \cup A^{0} \\
F^{\prime}\left(\beta_{j}^{(i)}\right)=y_{i}, & \forall j=1,2, \ldots, n_{i} & G^{\prime}\left(\alpha_{j}^{(i)}\right)=y_{i}, & \forall j=1,2, \ldots, m_{i} \\
& \text { e } i=1,2, \ldots & & \text { e } i=1,2, \ldots \\
0<F^{\prime}(x) \leq 1, & \forall x \in \mathbb{R} & 0<G^{\prime}(x) \leq 1, & \forall x \in \mathbb{R}
\end{array}
$$

Observamos que $F^{\prime}(x)<1, \forall x \in A^{-}$e $G^{\prime}(x)<1, \forall x \in A^{+}$. Considerando a função $H(x)=F(x)-G(x)$, temos:

- $\forall x \in A^{+}, H^{\prime}(x)=F^{\prime}(x)-G^{\prime}(x)=1-G^{\prime}(x)$, com $0<G^{\prime}(x)<1$. Logo, $H^{\prime}(x)>0$.

- $\forall x \in A^{-}, H^{\prime}(x)=F^{\prime}(x)-G^{\prime}(x)=F^{\prime}(x)-1$, com $0<F^{\prime}(x)<1$. Logo, $H^{\prime}(x)<0$.

- $\forall x \in A^{\circ}, H^{\prime}(x)=F^{\prime}(x)-G^{\prime}(x)=1-1=0$.

Podemos, desta forma, construir uma função $D N M(\mathbb{R})$ dependendo apenas da natureza dos subconjuntos enumeráveis tomados no teorema anterior.

Corolário 1.19. Existe uma função diferenciável em $\mathbb{R}$ que não é monótona em nenhum intervalo de $\mathbb{R}$.

Demonstração : Considere conjuntos $A^{+}, A^{-}, A^{o}$ que sejam densos em $\mathbb{R}$ e dois a dois disjuntos; por exemplo, podemos tomar $A^{+}=1+\mathbb{Q} \sqrt{2}, A^{-}=-1+\mathbb{Q} \sqrt{2}$ e $A^{0}=\mathbb{Q} \sqrt{2}$, sendo $a+\mathbb{Q} \sqrt{2}=\{a+q \cdot \sqrt{2} \mid a \in \mathbb{Q}, q \in \mathbb{Q}\}$. Usando o teorema 1.18, existe uma função $H$ que é diferenciável em $\mathbb{R}$ mas não monótona em nenhum intervalo do domínio. 
Podemos construir um espaço vetorial contido em $\mathcal{D} \mathcal{N} \mathcal{M}(\mathbb{R}) \cup\{0\}$ apenas escolhendo, convenientemente, os conjuntos densos enumeráveis $A^{+}, A^{-}$e $A^{0}$ do corolário 1.19 ; isto pode ser visto através do seguinte teorema:

Teorema 1.20. O conjunto $\mathcal{D N} \mathcal{M}(\mathbb{R})$ é lineável.

Demonstração : Consideremos a seqüência de ternas de conjuntos dois a dois disjuntos $\left\{A_{k}^{+}, A_{k}^{-}, A_{k}^{o}\right\}$ com as seguintes propriedades:

1) $A_{k}^{+}, A_{k}^{-}$e $A_{k}^{o}$ são enumeráveis e densos em $\mathbb{R}, \forall k \in \mathbb{N}$

2) $\left(A_{k}^{+} \cup A_{k}^{-} \cup A_{k}^{o}\right) \subset A_{k+1}^{o} \quad \forall k \in \mathbb{N}$

Um exemplo de seqüência destes conjuntos é a seguinte:

a) $\quad A_{k}^{+}=k+\mathbb{Q} \sqrt{2}, \forall k \in \mathbb{N}$

b) $\quad A_{k}^{-}=-k+\mathbb{Q} \sqrt{2}, \forall k \in \mathbb{N}$

c) $A_{1}^{0}=\frac{1}{2}+\mathbb{Q} \sqrt{2} ; \quad A_{k+1}^{0}=A_{k}^{0} \cup A_{k}^{-} \cup A_{k}^{+} \cup\left(k+\frac{1}{2}+\mathbb{Q} \sqrt{2}\right), \forall k \in \mathbb{N}$

Pelo corolário 1.19 , para cada $\mathrm{k}$, existe uma função $F_{k}$ definida e diferenciável em $\mathbb{R}$ e não monótona em intervalo nenhum tal que:
i) $F_{k}^{\prime}(x)>0$, se $x \in A_{k}^{+}$
ii) $F_{k}^{\prime}(x)<0$, se $x \in A_{k}^{-}$
iii) $F_{k}^{\prime}(x)=0, \quad$ se $\quad x \in A_{k}^{0}$

Pela construção destas funções, temos que $\left\{F_{k}\right\}_{k=1}^{\infty}$ é linearmente independente pois, para quaisquer escalares $\alpha_{1}, \alpha_{2}, \ldots, \alpha_{n}$, tais que $\sum_{k=1}^{n} \alpha_{k} \cdot F_{k} \equiv 0$, temos:

$$
0=\sum_{k=1}^{n} \alpha_{k} \cdot F_{k}^{\prime}(x), \forall x \in \mathbb{R}
$$

Tomando-se $x_{1} \in A_{1}^{+} \cup A_{1}^{-}$, temos que $x_{1} \in A_{k}^{0}, \forall k=2.3, \ldots, n$ e, com isso, $F_{k}^{\prime}\left(x_{1}\right)=0, \forall k=2,3, \cdots, n ; \operatorname{logo}, \alpha_{1} \cdot F_{1}^{\prime}\left(x_{1}\right)=0 \Longrightarrow \alpha_{1}=0$. Então, $\sum_{k=2}^{n} \alpha_{k} \cdot F_{k}(x)=0$, 
$\forall x \in \mathbb{R}$; tomando-se, agora, $x_{2} \in A_{2}^{+} \cup A_{2}^{-}$, obtemos, analogamente, $\alpha_{2} \cdot F_{2}^{\prime}\left(x_{2}\right)=0 \Longrightarrow$ $\alpha_{2}=0$. Continuando esse processo, podemos concluir que $\alpha_{3}=\alpha_{4}=\cdots=\alpha_{n}=0 \Longrightarrow$ $\left\{F_{k}\right\}_{k=1}^{\infty}$ é linearmente independente.

Resta mostrar que se $F(x)=\sum_{k=1}^{n} \alpha_{k} F_{k}(x)$ com $\alpha_{k}$ não nulos para $k=1,2, \cdots, n$, então $F$ é não monótona em nenhum intervalo de $\mathbb{R}, \forall n \in \mathbb{N}$. Como definido: $F_{1}^{\prime}(x)>0$, se $x \in A_{1}^{+}, F_{1}^{\prime}(x)<0$, se $x \in A_{1}^{-} \quad$ e $\quad F_{1}^{\prime}(x)=0$, se $x \in A_{1}^{0}$. Então, $\forall x \in\left(A_{1}^{+} \cup A_{1}^{-} \cup A_{1}^{0}\right), F_{k}^{\prime}(x)=0, \quad \forall k=2,3, \cdots, n$. Logo, $\quad F^{\prime}(x)=\alpha_{1} F_{1}^{\prime}(x)$, $\forall x \in\left(A_{1}^{+} \cup A_{1}^{-} \cup A_{1}^{0}\right)$. Suponhamos, sem perda de generalidade, que $\alpha_{1}>0$; então, $F^{\prime}(x)>0$, se $x \in A_{1}^{+}, F^{\prime}(x)<0$, se $x \in A_{1}^{-} \quad$ e $\quad F^{\prime}(x)=0$, se $x \in A_{1}^{0}$.

Desta forma, $F$ é não monótona em nenhum intervalo. Portanto, $\mathcal{D N} \mathcal{M}(\mathbb{R})$ é lineável. 



\section{Conjunto das Funções Fortemente Darboux}

O objetivo deste capítulo é provar que a lineabilidade (ver definição 1.1) do conjunto de todas as funções fortemente Darboux, que denotamos por $\mathcal{F} \mathcal{D}(\mathbb{R})$, é $2^{c}$. Os resultados estudados aqui foram baseados em [4].

\subsection{Funções de Darboux}

Até a segunda metade do século $X I X$, acreditava-se que toda função contínua $f: \mathbb{R} \longrightarrow \mathbb{R}$ era definida como uma função que tivesse a propriedade de assumir todos os valores entre quaisquer dois valores da sua imagem; em outras palavras, uma função tal que a imagem de qualquer intervalo do domínio era um intervalo ou um ponto.

Essa propriedade, atualmente, é conhecida como propriedade do valor intermediário ou propriedade de Darboux. Em 1875, Darboux mostrou que toda derivada tem a propriedade do valor intermediário e apresentou vários exemplos de derivadas descontínuas.

Por isso, funções que possuem esta propriedade são chamadas de funções de Darboux. Para considerações sobre este tema, citamos [7], [8] e suas referências.

De maneira mais geral, para definirmos uma função de Darboux, usamos o conceito 
de conjunto conexo cuja definição é a seguinte:

Definição 2.1. Seja $(X, \tau)$ um espaço topológico. Dizemos que $(X, \tau)$ é conexo se não existem abertos não vazios $U$ e $V$ tais que $U \cap V=\emptyset$ e $U \cup V=X$.

A partir disso, podemos definir funções de Darboux e fortemente Darboux.

\section{Definições 2.2. .}

i) Sejam $\left(X, \tau_{X}\right)$ e $\left(Y, \tau_{Y}\right)$ dois espaços topológicos. Uma função

$f:\left(X, \tau_{X}\right) \longrightarrow\left(Y, \tau_{Y}\right)$ é dita função de Darboux se, $\forall E \subset X$ conexo, $f(E) \subset Y$ é conexo.

ii) Uma função $f: \mathbb{R} \longrightarrow \mathbb{R}$ é dita fortemente Darboux se, para quaisquer $a, b \in \mathbb{R}, a<b$ e todo $y \in \mathbb{R}$, existe $x \in(a ; b)$ tal que $f(x)=y$.

No item i), se $X=Y=\mathbb{R}$ com a topologia usual, então esta definição é equivalente a propriedade do valor intermediário.

Podemos relacionar funções contínuas, de Darboux e fortemente Darboux através da seguinte proposição:

Proposição 2.3. Se $f$ é fortemente Darboux, então para toda função contínua $g: \mathbb{R} \longrightarrow \mathbb{R}, f+g$ é fortemente Darboux $\Longleftrightarrow f+g$ é de Darboux.

Demonstração : A implicação $(\Longrightarrow)$ é trivial. Para a outra implicação, temos:

Para qualquer intervalo limitado $I \subset \mathbb{R}$ aberto, $g$ é limitada. Por hipótese, $f(I)=\mathbb{R}$. Então, $f+g$ não é limitada nem inferiormente nem superiormente em $I$, caso contrário, deveríamos ter $|f(x)|<|g(x)|+|(f+g)(x)|<M$, para algum $M \in \mathbb{R}$ (absurdo); com isso, $\forall y \in \mathbb{R}$, existem $A, B \in \mathbb{R}$ tais que $A<y<B$ e $a, b \in I$ tais que $(f+g)(a)=A$ $\mathrm{e}(f+g)(b)=B$. Portanto, existe um $x$ entre $a$ e $b$ tal que $(f+g)(x)=y$ pois $(f+g)$ é de Darboux. Logo, $(f+g)(I)=\mathbb{R}$. 
A hipótese da função $g$ ser contínua na proposição anterior é essencial uma vez que, se tomarmos, por exemplo, $g \equiv-f$ (sendo $f$ uma função fortemente Darboux, $-f$ também o será e, portanto, totalmente descontínua, ou seja, sem nenhum ponto de continuidade), obtemos $f+g \equiv 0$ ( função identicamente nula) e, por conseguinte, $f+g$ é uma função de Darboux, mas não é fortemente Darboux. Outros resultados sobre funções fortemente Darboux podem ser vistos, por exemplo, em [9] no capítulo 7.

De acordo com as definições 2.2 itens i) e ii), podemos concluir que toda função fortemente Darboux é de Darboux. Agora, nem toda função de Darboux é contínua; abaixo, apresentamos dois contra-exemplos que comprovam esse fato:

\section{Exemplos 2.4. .}

i) Seja $f: \mathbb{R} \longrightarrow \mathbb{R}$ uma função definida por:

$$
f(x)=\left\{\begin{array}{r}
x^{2} \cdot \sin \left(\frac{1}{x}\right), \text { se } x \neq 0 \\
0, \text { se } x=0
\end{array}\right.
$$

Desta forma, a derivada $f^{\prime}$ de $f$ é contínua em todos os pontos do domínio, exceto para $x=0$; no entanto, como demonstrou Darboux, $f^{\prime}$ é uma função de Darboux.

\section{ii) Função de Lebesgue}

Uma função de Darboux pode ser totalmente descontínua, ou seja, não ter nenhum ponto de continuidade. H. Lebesgue [17], pg. 97, foi, provavelmente, o primeiro matemático a apresentar um exemplo de uma função de Darboux totalmente descontinua com a seguinte propriedade:

L: $\quad$ Para $f:[0 ; 1] \longrightarrow[0 ; 1]$ e para quaisquer $a, b \in[0 ; 1], a<b$ e todo $y \in[0 ; 1]$, existe um $x \in(a ; b)$ tal que $f(x)=y$.

Antes de apresentarmos a função de Lebesgue, ressaltamos que ela pode ser considerada como uma função fortemente Darboux se restringirmos o dominio e a imagem para $[0 ; 1]$; segue, aqui, sua descrição:

Seja $x$ um número real arbitrário em $[0 ; 1]$ escrito na forma de sua expansão decimal $x=0, a_{1} a_{2} a_{3} \ldots$. Se $x$ é um número racional, vamos escrevê-lo sempre na forma 
expandida de dízima periódica (poderia ser feita analogamente se considerássemos $x$ escrito na forma decimal com um número finito de dígitos não nulos). Vamos estabelecer o valor de $f(x)$ dependendo se $0, a_{1} a_{3} a_{5} \ldots$ é ou não um número racional. Os dígitos de $x$ com índices pares ficam reservados para os dígitos da imagem de $x$ pela $f$ quando escrita na forma de expansão decimal.

Para qualquer $x=0, a_{1} a_{2} a_{3} \ldots \in[0 ; 1]$, considere o número $z=0, a_{1} a_{3} a_{5} \ldots e$ vamos definir a função $f:[0 ; 1] \longrightarrow[0 ; 1]$ em termos de $z$ como:

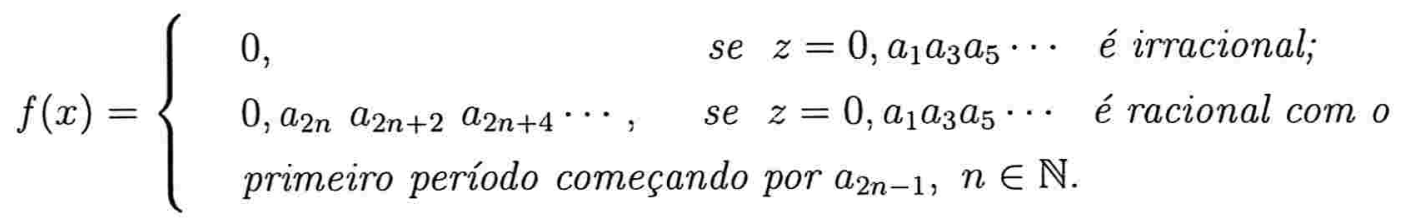

Como definida acima, $f$ possui a propriedade $L$. De fato, para qualquer intervalo aberto $I \subset[0 ; 1]$ dado, podemos tomar $n \in \mathbb{N}$ suficientemente grande e escolher dígitos $a_{1}, a_{2}, \cdots, a_{2 n-1}$ tais que $I$ contenha $0, a_{1} a_{2} \cdots a_{2 n-1}$ e todos os números da forma $0, a_{1} a_{2} \cdots a_{2 n-1} a_{2 n} \cdots$ começando sempre pelos mesmos $2 n-1$ primeiros dígitos.

Seja, agora, $y=0, b_{1} b_{2} b_{3} \cdots \in[0 ; 1]$ arbitrário. Vamos mostrar que existe $x \in I$ tal que $f(x)=y$. Para isso, escolhemos dígitos $a_{2 n+1}, a_{2 n+3}, a_{2 n+5}, \cdots$ convenientemente tais que $0, a_{1} a_{3} a_{5} \cdots a_{2 n-1} a_{2 n+1} a_{2 n+3} \cdots$ seja periódico com primeiro período começando por $a_{2 n-1}$; temos, desta forma, que o número procurado é $x=0, a_{1} a_{2} a_{3} \cdots a_{2 n-1} b_{1} a_{2 n+1} b_{2} a_{2 n+3} \cdots$ e $f(x)=y$.

Observemos que $f$ definida assim é descontínua em todos os pontos do domínio. O gráfico $G_{f}=\{(x ; f(x)) \mid x \in[0 ; 1]\}$ é denso no quadrado unitário, ou seja, $\bar{G}_{f}=[0 ; 1] \times[0 ; 1]$ embora cada segmento vertical $\{x\} \times[0 ; 1]$ encontre $G_{f}$ em apenas um ponto.

Como o objetivo deste capítulo é provar que a lineabilidade do conjunto de todas as funções fortemente Darboux é $2^{c}$, vamos, primeiramente, apresentar um exemplo de uma função $f: \mathbb{R} \longrightarrow \mathbb{R}$ desse tipo. Para tal, vamos precisar usar, entre outros conceitos, o conjunto de Cantor e a função de Cantor que descrevemos na próxima seção. 


\subsection{O Conjunto de Cantor e a Função de Cantor}

Nesta seção, vamos introduzir o conjunto de Cantor apresentando algumas propriedades relativas ao seu "tamanho" e a função de Cantor que será largamente usada quando apresentarmos o exemplo de uma função fortemente Darboux já mencionada.

\section{Conjunto de Cantor $C$}

O conjunto de Cantor $C$ é obtido, a partir do intervalo fechado $[0 ; 1]$, através de uma seqüência de eliminações de intervalos abertos como indicado nas etapas abaixo:

$1^{o}$ ) Eliminação do intervalo aberto $\left(\frac{1}{3} ; \frac{2}{3}\right)$ depois de dividir o intervalo $[0 ; 1]$ em três subintervalos de mesmo comprimento.

$2^{o}$ ) Cada um dos intervalos fechados restantes $\left[0 ; \frac{1}{3}\right]$ e $\left[\frac{2}{3} ; 1\right]$ é dividido em três subintervalos de mesmo comprimento eliminando-se seus intervalos abertos centrais $\left(\frac{1}{9} ; \frac{2}{9}\right)$ e $\left(\frac{7}{9} ; \frac{8}{9}\right)$

$\left.3^{o}\right)$ Cada um dos intervalos fechados restantes $\left[0 ; \frac{1}{9}\right],\left[\frac{2}{9} ; \frac{1}{3}\right],\left[\frac{2}{3} ; \frac{7}{9}\right]$ e $\left[\frac{8}{9} ; 1\right]$ é dividido em três subintervalos também de mesmo comprimento eliminando-se seus intervalos abertos centrais $\left(\frac{1}{27} ; \frac{2}{27}\right),\left(\frac{7}{27} ; \frac{8}{27}\right),\left(\frac{19}{27} ; \frac{20}{27}\right)$ e $\left(\frac{25}{27} ; \frac{26}{27}\right)$.

$4^{\circ}$ ) Continuando este processo indefinidamente, o conjunto de Cantor $C$ é o complementar da união de todos os intervalos abertos retirados, isto é, $C=[0 ; 1] \backslash \bigcup_{n=1}^{\infty} I_{n}$ com $I_{1}, I_{2}, \cdots, I_{n}, \cdots$ os intervalos abertos removidos.

Antes de apresentarmos algumas propriedades do conjunto de Cantor, vamos usar as seguintes definições:

Definição 2.5. Um conjunto $A \subset \mathbb{R}$ é dito de medida nula se, para todo $\epsilon>0$, existe uma famitia enumerável de intervalos abertos $\left\{I_{i}=\left(x_{i} ; y_{i}\right): i \in \mathbb{N}\right\}$ tal que $A \subset \bigcup_{i=1}^{\infty} I_{i} \quad e \sum_{i=1}^{\infty}\left|y_{i}-x_{i}\right|<\epsilon$.

Definição 2.6. Um conjunto $A \subset \mathbb{R}$ é dito raro se todo intervalo aberto tem subintervalo disjunto de A ou, equivalentemente, se o interior do seu fecho é vazio. 
Podemos, agora, estabelecer a seguinte proposição:

Proposição 2.7. Existe uma bijeção entre o conjunto de Cantor $C$ e o intervalo [0;1]; além disso, o conjunto $C$ é perfeito, raro e de medida nula.

Demonstração : Para demonstrarmos essa proposição, lembramos que um conjunto é perfeito se é fechado e todos os seus pontos são de acumulação, ou seja, um conjunto perfeito não tem pontos isolados.

Primeiramente, na construção de $C$, foram removidos intervalos abertos e somente eles; a união desses intervalos é um conjunto aberto; o conjunto $C$, sendo o complementar deste conjunto aberto dentro do intervalo fechado $[0 ; 1]$, é, portanto, fechado.

Denotemos por pontos extremos de um intervalo (fechado, aberto ou semi-aberto) aos pontos da fronteira deste intervalo. Os pontos extremos de cada intervalo aberto eliminado na construção de $C$ ( por exemplo $\frac{1}{9}, \frac{2}{9}, \frac{1}{3}, \frac{2}{3}$, etc.) não foram removidos; logo, eles pertencem ao conjunto $C$; já sabemos que $C$ é fechado e, com isso, os pontos de acumulação dos pontos extremos também estão em $C$. Por exemplo, na construção do conjunto $C$ por etapas como descritas anteriormente, partindo-se de $\frac{1}{3}$, na segunda etapa de intervalos abertos removidos, o ponto extremo mais próximo é $\frac{2}{9}=\frac{1}{3}-\frac{1}{9}$; esse, por sua vez, na etapa seguinte, tem um ponto extremo mais próximo igual a $\frac{7}{27}=\frac{1}{3}-\frac{1}{9}+\frac{1}{27}$; continuando indefinidamente este processo, obtemos uma seqüência de pontos extremos cujo ponto de acumulação é $\frac{1}{3}-\frac{1}{9}+\frac{1}{27}-\cdots=\frac{1}{4}$. Portanto, há pontos de acumulação que não são pontos extremos dos intervalos removidos. O conjunto de Cantor $C$ é formado pelos pontos extremos de todos os intervalos removidos e pelos pontos de acumulação como descritos acima.

Uma característica interessante em relação aos números pertencentes ao conjunto $C$ é que, quando escritos na base 3, podem ser representados sem conter o algarismo 1 entre seus dígitos. Por exemplo, todo número escrito na forma de expansão ternária cujo primeiro dígito após a vírgula é 1 está entre $\frac{1}{3}$ e $\frac{2}{3}$ (inclusive) e que está contido no primeiro intervalo fechado cujo interior foi removido na construção de $C$. Já os números do intervalo $\left[0 ; \frac{1}{3}\right]$ começam com o dígito 0 após a vígula, enquanto que, para o intervalo $\left[\frac{2}{3} ; 1\right]$, começam com o dígito 2 . Devemos ressaltar que $\frac{1}{3}=(0,1000 \cdots)_{3}=(0,0222 \cdots)_{3}$ 
e $\frac{2}{3}=(0,2000 \cdots)_{3}=(0,1222 \cdots)_{3}$ e, portanto, são números em $C$ e que podem ser representados sem conter o dígito 1 . Já para o intervalo $\left[0 ; \frac{1}{3}\right]$, todos os números entre $\frac{1}{9}=(0,0100 \cdots)_{3}=(0,00222 \cdots)_{3}$ e $\frac{2}{9}=(0,02000 \cdots)_{3}=(0,01222 \cdots)_{3}$ apresentam o segundo dígito, quando escrito na sua forma de expansão ternária, igual a 1. E, mais uma vez, foram removidos, exceto seus pontos extremos. Essa observação vale, analogamente, para todos os demais pontos que possuem uma representação com somente 0 e 2 entre seus dígitos.

Como cada número pertencente ao conjunto $C$ pode ser representado somente por dígitos 0 e 2 quando escritos na base 3, podemos dividir cada dígito por 2 obtendo um novo número formado somente por $0^{\prime} s$ e $1^{\prime} s$. Esse novo número passa ser, agora, interpretado na base 2 . Por exemplo, $\frac{1}{3}=(0,0222 \cdots)_{3}$ nos fornece o número $\frac{1}{2}=(0,0111 \cdots)_{2}$.

Esta correspondência não é injetora pois, por exemplo, $\frac{2}{3}=(0,2000 \cdots)_{3}$ fornece também o número $\frac{1}{2}=(0,1000 \cdots)_{2}$. Isto ocorre para todos os extremos dos intervalos removidos e somente para eles. Podemos, portanto, concluir que existe uma correspondência biunívoca entre o conjunto de Cantor $C$, exceto por um conjunto enumerável formado por um dos pontos extremos de cada intervalo removido e o conjunto de todos os números reais, exceto por um conjunto enumerável formado por um dos números que possuam duas representações na base 2. Por meio de uma correspondência biunívoca entre estes dois conjuntos enumeráveis, obtemos uma bijeção entre o conjunto de Cantor e o intervalo $[0 ; 1]$.

Para mostrar que $C$ é um conjunto perfeito basta mostrar que cada um de seus pontos é de acumulação. Logo, basta mostrar que os pontos extremos são de acumulação. De fato, consideremos, por exemplo, o ponto $\frac{1}{3}$; à sua esquerda, há um intervalo $\left[0 ; \frac{1}{3}\right]$ de comprimento $\frac{1}{3}$; deste intervalo, removemos o intervalo $\left(\frac{1}{9} ; \frac{2}{9}\right)$ restando o intervalo $\left[\frac{2}{9} ; \frac{1}{3}\right]$ adjacente ao ponto $\frac{1}{3}$. O seu comprimento é, portanto, igual a $\frac{1}{9}$. Repetindo este procedimento, o próximo intervalo adjacente a $\frac{1}{3}$ tem comprimento igual a $\frac{1}{27}$. Podemos continuar indefinidamente este processo; para qualquer vizinhança de $\frac{1}{3}$, existe um inter- 
valo fechado de comprimento, $\frac{1}{3^{n}}$, para algum $n \in \mathbb{N}$ suficientemente grande, adjacente a $\frac{1}{3}$ e que não foi removido até uma certa etapa. Esse intervalo, por sua vez, contém um ponto extremo diferente de $\frac{1}{3}$ e que pertence ao conjunto $C$. Logo, $\frac{1}{3}$ é ponto de acumulação. Argumentação similar pode ser feita para qualquer outro ponto extremo.

Disso, concluímos que $C$ é fechado com todos os seus pontos sendo de acumulação. $C$ é perfeito.

Vamos, agora, mostrar que $C$ é raro; para qualquer intervalo aberto $I$ contido em $[0 ; 1]$ de comprimento $m>0$, podemos determinar $n \in \mathbb{N}$ tal que $\frac{1}{3^{n}}<m$ após a n-ésima etapa na construção de $C$ como descrita anteriormente de modo que $I$ contenha um intervalo fechado de comprimento $\frac{1}{3^{n}}$; desse intervalo fechado, removido o intervalo central aberto, concluímos que existe um aberto contido em $I$ e que não contém nenhum ponto de $C$; como $I$ foi tomado arbitrariamente, temos que $C$ é raro.

Após a n-ésima etapa, o comprimento total dos intervalos fechados não removidos é igual a $\left(\frac{2}{3}\right)^{n}$; com isso, a medida do conjunto de Cantor $C$ é igual $\lim _{n \rightarrow \infty}\left(\frac{2}{3}\right)^{n}=0$. Portanto, o conjunto de Cantor $C$ tem medida nula.

\section{Função de Cantor $\Phi$}

Agora temos todos os requisitos para definir a função $\Phi$ de Cantor:

Para todo $\quad x=\left(0, a_{1} a_{2} a_{3} \cdots\right)_{3} \in C$ com $a_{n}=0$ ou $2, \quad n=1,2, \cdots$, seja

$$
\Phi(x)=\left(0, \frac{a_{1}}{2} \frac{a_{2}}{2} \frac{a_{3}}{2} \cdots\right)_{2} .
$$

Pode-se verificar que, para quaisquer $x_{1}, x_{2} \in C, \quad x_{1}<x_{2}$ temos:

$$
\Phi\left(x_{1}\right)=\Phi\left(x_{2}\right) \Longleftrightarrow x_{1}=\left(0, a_{1} a_{2} a_{3} \cdots a_{n} 0222 \cdots\right)_{3} \text { e } x_{2}=\left(0, a_{1} a_{2} a_{3} \cdots a_{n} 200 \cdots\right)_{3} .
$$

Em outras palavras, $\Phi\left(x_{1}\right)=\Phi\left(x_{2}\right)$ se, e somente se, $x_{1}$ e $x_{2}$ são pontos extremos de um mesmo intervalo aberto removido.

Por fim, definimos, para todo $x \in[0 ; 1] \backslash C, \Phi(x)=\Phi\left(x_{1}\right)=\Phi\left(x_{2}\right)$ para o intervalo aberto removido $\left(x_{1} ; x_{2}\right)$ ao qual $x$ pertence, ou seja, $\Phi$ é constante no fecho de cada intervalo aberto removido. 
Pode-se verificar, então, que $\Phi$ é não decrescente, contínua com derivada $\Phi^{\prime}=0$, $\forall x \in[0 ; 1] \backslash C$ ( não existe $\Phi^{\prime}(x), \forall x \in C$ ).

Para mais informações e propriedades relativas ao conjunto e à função de Cantor ver, por exemplo, [5] e [13].

\subsection{Função Fortemente Darboux}

Vamos começar esta seção apresentando uma função fortemente Darboux e, a partir dela, atingirmos o objetivo deste capítulo que é exibir um espaço vetorial de dimensão $2^{c}$ em $\mathcal{F D}(\mathbb{R}) \cup\{0\}$. Vamos descrevê-la por etapas:

1) Definimos uma função $g:(0 ; 1) \longrightarrow \mathbb{R}$ dada por:

$$
g(x)=\operatorname{tg}\left[\pi\left(\Phi(x)-\frac{1}{2}\right)\right]
$$

sendo $\Phi$ a função de Cantor; então, para o conjunto de Cantor $C$, temos $g[C \cap(0 ; 1)]=\mathbb{R}$.

2) Para qualquer intervalo aberto $I=(a ; b)$, definimos o seguinte conjunto:

$$
C_{I}=\{a+(b-a) x: x \in C \cap(0 ; 1)\} \subset I
$$

Não é difícil observar que o conjunto $C_{I}$, como foi definido, tem medida nula.

3) Definimos uma função sobrejetora $g_{I}: C_{I} \longrightarrow \mathbb{R}$ dada por:

$$
g_{I}(x)=g\left(\frac{x-a}{b-a}\right)
$$

4) Finalmente, definimos a função $f: \mathbb{R} \longrightarrow \mathbb{R}$ como segue:

a) Para qualquer $x \in \mathbb{Z}, f(x)=0$. Definimos, agora, a seqüência $\left(U_{n}\right)_{n \in \mathbb{N}}$ de conjuntos abertos da seguinte maneira: $U_{1}=\mathbb{R} \backslash \mathbb{Z}$, ou seja, $U_{1}$ é a união de todos os 
intervalos abertos da forma $(n ; n+1), \forall n \in \mathbb{Z}$; para cada um desses intervalos, que denotamos de maneira geral por $I$, seja $C_{I}$ o conjunto de medida nula como descrito acima.

b) Para cada $x$ pertencente a cada um desses conjuntos $C_{I}$, definimos $f(x)=g_{I}(x)$ e denotemos por $D_{1}$ o conjunto formado por todos esses pontos; como definido, podemos concluir que $D_{1}$ tem medida nula. Seja $U_{2} \subset U_{1}$ o subconjunto onde a $f$ ainda não foi definida. Logo, como $U_{2}$ é um conjunto aberto, podemos escrevê-lo como união disjunta de intervalos abertos. Para cada um desses intervalos $I$ de $U_{2}$ seja $C_{I}$ o conjunto de medida nula como descrito no item 2).

c) Para cada $x$ pertencente a cada um desses conjuntos $C_{I}$, definimos $f(x)=g_{I}(x)$ e denotemos por $D_{2}$ o conjunto dos pontos $x$ tais que vale essa última igualdade; como definido, podemos concluir que $D_{2}$ tem medida nula.

d) Seja $U_{3} \subset U_{2}$ o subconjunto onde a $f$ ainda não foi definida. Para esse subconjunto executamos, de maneira análoga, os passos 4b) e 4c) descritos acima. Efetuamos o mesmo processo para cada elemento da seqüência $\left(U_{n}\right)_{n \in \mathbb{N}} \operatorname{com} U_{n+1} \subset U_{n}$, $\forall n \in \mathbb{N}$. A função $f$ fica, então, definida no conjunto de medida nula $\bigcup_{n \in \mathbb{N}} D_{n}$.

e) Por fim, definimos a $f$ igual a zero para todos os demais pontos onde ela ainda não foi definida. (Por exemplo, todos os pontos da forma $n+\frac{1}{2}, \forall n \in \mathbb{N}$ ). Desta forma, a função $f$ é identicamente nula exceto num conjunto de medida nula.

A função $f$ assim definida é fortemente Darboux; de fato, para qualquer intervalo aberto $J \subset \mathbb{R}$, existe um $n \in \mathbb{N}$ suficientemente grande tal que $U_{n}$ contém um dos intervalos abertos $I$ descritos acima com $I \subseteq U_{n} \cap J$. Portanto, tomando-se o subconjunto $C_{I} \subset I$, temos $f\left(C_{I}\right)=f(J)=\mathbb{R}$.

Nosso objetivo, agora, é apresentar o principal resultado desse capítulo que é verificar que a lineabilidade do conjunto $\mathcal{F} \mathcal{D}(\mathbb{R})$ é $2^{c}$. Para isso, vamos, primeiramente, verificar que podemos obter un espaço vetorial contido em $\mathcal{F} \mathcal{D}(\mathbb{R}) \cup\{0\}$ usando compostas de funções sobrejetoras com uma função fortemente Darboux fixada. Para atingirmos esses objetivos, vamos precisar de alguns resultados prévios; o primeiro deles é um lema que será usado em todos os demais resultados deste capítulo. 
Lema 2.8. Sejam $C_{1}, C_{2}, \cdots, C_{m} m$ subconjuntos não vazios de $\mathbb{R}$ e distintos dois a dois. Então, existe $k \in\{1,2, \cdots, m\}$ tal que $C_{k} \backslash C_{i} \neq \emptyset, \forall i \neq k$.

Demonstração : A demonstração é feita por indução sobre $m$ :

- Para $m=2$, seja $C_{1} \neq C_{2}$; sem perda de generalidade, podemos tomar $k=2$ observando que $C_{2} \backslash C_{1} \neq \emptyset$.

- Suponhamos válido para $m-1$ subconjuntos. Então, sem perda de generalidade, podemos assumir $k=m-1$ e obtermos $C_{m-1} \backslash C_{i} \neq \emptyset, \forall i \in\{1,2, \cdots, m-2\}$ ou, de outra forma, existem $x_{i} \in \mathbb{R}$ tais que $x_{i} \in C_{m-1} \backslash C_{i}, \forall i \in\{1,2, \cdots, m-2\}$.

- Vamos provar para $m$ subconjuntos não vazios distintos dois a dois:

Analisemos em relação à $C_{m-1}$. Há duas possibilidades:

1) Se $C_{m-1} \not \subset C_{m}$, então existe $x_{m} \in \mathbb{R}$ tal que $x_{m} \in C_{m-1} \backslash C_{m}$. Logo, para esse caso, podemos tomar $k=m-1$.

2) Se $C_{m-1} \subset C_{m}$, então existe $x_{m-1} \in \mathbb{R}$ tal que $x_{m-1} \in C_{m} \backslash C_{m-1}$. Logo, $x_{i} \in$ $C_{m} \backslash C_{i}, \forall i \in\{1,2, \cdots, m-1\}$ e podemos tomar $k=m$; o lema está demonstrado.

Vamos aplicar este lema para mostrar que existe um espaço vetorial contido no conjunto formado pela função identicamente nula e por todas as funções sobrejetoras $f: \mathbb{R}^{\mathbb{N}} \longrightarrow \mathbb{R}$.

Proposição 2.9. Existe um subespaço vetorial $S$ contido em $\mathcal{F}\left(\mathbb{R}^{\mathbb{N}} ; \mathbb{R}\right)$ com as seguintes propriedades:

a) Toda função não nula de $S$ é sobrejetora.

b) $\operatorname{dim} S=2^{c}$, sendo $c$ a cardinalidade de $\mathbb{R}$.

Demonstração : Sejam $0 \neq r \in \mathbb{R}$ fixado e um conjunto não vazio $C \subset \mathbb{R}$ arbitrário. Definimos uma função $H_{C}: \mathbb{R}^{\mathbb{N}} \longrightarrow \mathbb{R}$ como:

$$
H_{C}\left(y, x_{1}, x_{2}, \cdots\right)=\varphi_{r}(y) \cdot \prod_{i=1}^{\infty} \chi_{C}\left(x_{i}\right)
$$


sendo $\varphi_{r}: \mathbb{R} \longrightarrow \mathbb{R}$ dada por $\varphi_{r}(y)=e^{r y}-e^{-r y}$ e $\chi_{C}$ a função característica de $C$.

Não é difícil ver que a $\varphi_{r}$ é sobrejetora. Afirmamos que $H_{C}$ também é sobrejetora. De fato, $\forall s \in \mathbb{R}$, escolhemos $a \in \mathbb{R}$ tal que $\varphi_{r}(a)=s$ e, tomando-se $x=(a, b, b, b, \cdots) \in \mathbb{R}^{\mathbb{N}}$ para algum $b \in C$, temos $H_{C}(x)=s$.

Seja $A:=\left\{H_{C}: \emptyset \neq C \subset \mathbb{R}\right\}$ um conjunto de funções sobrejetoras como definidas acima; vamos denotar por $S$ o espaço vetorial gerado por $A$, ou seja, $S:=[A]$ e verificar que $S$ satisfaz as condições da proposição:

Seja $m \in \mathbb{N}$ arbitrário; tomemos, então, $C_{1}, C_{2}, \cdots, C_{m} m$ subconjuntos de $\mathbb{R}$ não vazios e distintos dois a dois. Sejam $\lambda_{1}, \cdots, \lambda_{m}$ escalares tais que $\sum_{j=1}^{m} \lambda_{j} H_{C_{j}} \equiv 0(*)$ com $H_{C_{j}} \in A, \forall j$. Como $C_{i} \neq C_{j}$ para $i \neq j$, podemos assumir, sem perda de generalidade, que, $\forall j<m$, existam $x_{j} \in \mathbb{R}$ tais que $x_{j} \in C_{m} \backslash C_{j}$ (lema anterior). Para $x=\left(1, x_{1}, x_{2}, \cdots, x_{m-2}, x_{m-1}, x_{m-1}, x_{m-1} \cdots\right) \in \mathbb{R}^{\mathbb{N}}$, obtemos:

$$
0=\sum_{j=1}^{m} \lambda_{j} H_{C_{j}}(x)=\varphi_{r}(1) \cdot \sum_{j=1}^{m}\left[\lambda_{j} \cdot \prod_{i=1}^{m-2} \chi_{C_{j}}\left(x_{i}\right) \cdot \prod_{i=m-1}^{\infty} \chi_{C_{j}}\left(x_{m-1}\right)\right]=\varphi_{r}(1) \cdot \lambda_{m}
$$

Logo, $\lambda_{m}=0$ pois $\varphi_{r}(1) \neq 0$. Desta forma, temos de $(*)$ que $\sum_{j=1}^{m-1} \lambda_{j} H_{C_{j}} \equiv 0$.

Agora, assumindo que $\forall j<m-1$ existam $y_{j} \in \mathbb{R}$ tais que $y_{j} \in C_{m-1} \backslash C_{j}$ e considerando $y=\left(1, y_{1}, y_{2}, \cdots, y_{m-3}, y_{m-2}, y_{m-2}, \cdots\right)$, obtemos $\lambda_{m-1}=0$.

Continuando esse processo, uma vez que é finito, obtemos $\lambda_{m-2}=\lambda_{m-3}=\cdots=$ $=\lambda_{1}=0$. Logo, $\left\{H_{C_{1}}, \cdots, H_{C_{m}}\right\} \subset A$ é linearmente independente.; como esse conjunto foi tomado arbitrariamente em $A$, concluímos que $A$ é linearmente independente. Usando; sem perda de generalidade, os mesmos conjuntos $C_{1}, C_{2}, \cdots, C_{m}$, vamos, agora, verificar que $\forall h \in S \backslash\{0\}$ com $h \equiv \sum_{j=1}^{m} a_{j} H_{C_{j}}, a_{j} \in \mathbb{R} \backslash\{0\}$, h é sobrejetora:

Dado $s \in \mathbb{R}$ arbitrário, escolhemos $a \in \mathbb{R}$ tal que $\varphi_{r}(a)=\frac{s}{a_{m}}$. Então, tomando-se $z=\left(a, x_{1}, x_{2}, \cdots, x_{m-2}, x_{m-1}, x_{m-1}, \cdots\right) \in \mathbb{R}^{\mathbb{N}}$ com $x_{j} \in C_{m} \backslash C_{j}$ como apresentados anteriormente, temos: 


$$
h(z)=\sum_{j=1}^{m} a_{j} H_{C_{j}}(z)=\varphi_{r}(a) \cdot \sum_{j=1}^{m}\left[a_{j} \cdot \prod_{i=1}^{m-2} \chi_{C_{j}}\left(x_{i}\right) \cdot \prod_{i=m-1}^{\infty} \chi_{C_{j}}\left(x_{m-1}\right)\right]=\frac{s}{a_{m}} \cdot a_{m}=s
$$

Desta forma, h é sobrejetora. Resta mostrar que a cardinalidade de $A$ é $2^{c}$ para a conclusão dessa proposição. Mas isso segue diretamente do fato que o conjunto das partes de $\mathbb{R}$ tem cardinalidade $2^{c}$ e, portanto, $\operatorname{dim} S=2^{c}$.

Estamos interessados, agora, em encontrar um espaço vetorial contido no conjunto formado pela função identicamente nula e por todas as funções sobrejetoras $f: \mathbb{R} \longrightarrow \mathbb{R}$. Para isso, vamos usar a proposição anterior juntamente com o fato de existir uma bijeção de $\mathbb{R}^{\mathbb{N}}$ em $\mathbb{R}$ que descreveremos a seguir. Antes de apresentarmos um resultado que nos garanta essa bijeção, vamos introduzir a seguinte definição:

Definição 2.10. Dados dois conjuntos $A$ e $B$, dizemos que $A$ e $B$ possuem a mesma cardinalidade ou que $A$ é equipotente a $B$ e indicamos por $A \equiv B$ se existe uma função bijetora entre eles.

Posto isso, de acordo com o que foi exposto sobre o conjunto de Cantor, podemos concluir que a cardinalidade do conjunto $C$ é igual a do intervalo $[0 ; 1]$ cujo valor é $c$. Logo, $C$ é um conjunto não enumerável; na verdade, esta conclusão já poderia ter sido obtida pelo fato de que o conjunto de Cantor é perfeito e não vazio (aplicação do teorema da categoria de Baire).

O teorema seguinte nos permite dizer que não é necessário exibir uma função bijetora para mostrar que dois conjuntos sejam equipotentes:

Teorema 2.11. (Cantor-Schröder-Bernstein): Dados dois conjuntos $X$ e $Y$, se $X$ é equipotente a uma parte de $Y$ e $Y$ é equipotente a uma parte de $X$, então $X$ e $Y$ são equipotentes.

Demonstração : Ver, por exemplo, [1], pg. 8-9. 
Este teorema nos diz que se apresentarmos uma função injetora de $X$ em $Y$ e uma outra injeção de $Y$ em $X$, a equipotência entre estes conjuntos fica configurada. Um importante exemplo de aplicação deste teorema para esse trabalho é o seguinte:

Exemplo 2.12. $\mathbb{R}^{\mathbb{N}} \equiv \mathbb{R}$

Demonstração : Consideremos a função $f: \mathbb{R} \longrightarrow(0,1)$ definida por:

$$
f(x)=\frac{2^{x}}{1+2^{x}}, \quad \forall x \in \mathbb{R}
$$

Como $f$ é bijetora, temos $\mathbb{R} \equiv(0,1)$. Afirmamos que, $\mathbb{R}^{\mathbb{N}} \equiv(0,1)^{\mathbb{N}} ;$ de fato, tomandose a função $\Psi: \mathbb{R}^{\mathbb{N}} \longrightarrow(0,1)^{\mathbb{N}}$ dada por

$$
\Psi\left(x_{1}, x_{2}, \cdots\right)=\left(f\left(x_{1}\right), f\left(x_{2}\right), \cdots\right),
$$

sendo $f$ como definida acima, temos que $\Psi$ é bijetora.

Seja a função $G:(0,1) \longrightarrow(0,1)^{\mathbb{N}}$ definida por $G(x)=(x, 0,0, \cdots)$.

Como definida, $G$ é injetora. Resta, então, encontrar uma função injetora $F:(0,1)^{\mathbb{N}} \longrightarrow(0,1)$. Para isso, lembremos que $(0,1)^{\mathbb{N}} \equiv\left\{\left(x_{n}\right)_{n \geq 0}: x_{n} \in(0,1), \forall n \in \mathbb{N}_{0}\right\}$.

Consideremos $x_{n}=0, x_{0}^{n} x_{1}^{n} \cdots x_{k}^{n} \cdots$, a expansão binária com $\left\{k: x_{k}^{n}=1\right\}$ sendo um conjunto infinito.

Vamos definir a função $h: \mathbb{N} \longrightarrow \mathbb{N}_{0} \times \mathbb{N}_{0}$ por $h(m)=(n, k)$, com $m=2^{n} \cdot(2 k+1)$.

Certamente, $h$ é bijetora; finalmente podemos definir a função $F \operatorname{como} F(x)=z$, sendo $x=\left(x_{n}\right)_{n \geq 0}$ e $z=0, z_{1} z_{2} z_{3} \cdots z_{m} \cdots \operatorname{com} z_{m}=x_{k}^{n}$ e $(n, k)=h(m)$.

Para verificarmos que $F$ é injetora, tomemos $x=\left(x_{n}\right)_{n}, y=\left(y_{n}\right)_{n} \in(0,1)^{\mathbb{N}}, x \neq y$; com isso, existe $n \in \mathbb{N}$ tal que $x_{n} \neq y_{n}$ sendo $x_{n}=0, x_{0}^{n} x_{1}^{n} \cdots x_{k}^{n} \cdots$ e $y_{n}=0, y_{0}^{n} y_{1}^{n} \cdots y_{k}^{n} \cdots$. Mas isso implica que existe $k \in \mathbb{N}$ tal que $x_{k}^{n} \neq y_{k}^{n}$; como $F(x)=z=0, z_{1} z_{2} z_{3} \cdots z_{m} \cdots$ e $F(y)=\bar{z}=0, \bar{z}_{1} \bar{z}_{2} \bar{z}_{3} \cdots \bar{z}_{m} \cdots$, temos que $x_{k}^{n}=z_{m} \neq \bar{z}_{m}=y_{k}^{n} \Longrightarrow F(x) \neq F(y)$.

Logo, $F$ é injetora.

O corolário seguinte é uma conseqüência direta deste exemplo e da proposição anterior: 
Corolário 2.13. Existe um subespaço vetorial $V$ contido em $\mathcal{F}(\mathbb{R} ; \mathbb{R})$ com as seguintes propriedades:

a) Toda função não nula de $V$ é sobrejetora.

b) $\operatorname{dim} V=2^{c}$.

Demonstração : Sabemos que existe uma função bijetora $h: \mathbb{R} \longrightarrow \mathbb{R}^{\mathbb{N}}$. Logo, podemos tomar a função $H: \mathbb{R} \stackrel{h}{\longrightarrow} \mathbb{R}^{\mathbb{N}} \stackrel{H_{C}}{\longrightarrow} \mathbb{R}$ que é sobrejetora uma vez que $H=H_{C} \circ h$ (composta de sobrejetoras). Para o conjunto $A$ definido na proposição anterior, sejam $B:=\left\{H_{C} \circ h: \mathbb{R} \longrightarrow \mathbb{R} \mid H_{C} \in A\right\}$ e $V:=[B]$. Agora, para provar que $B$ é linearmente independente e que gera um espaço de funções sobrejetoras, usamos o mesmo raciocínio da proposição 2.9. Basta, então, verificar que a cardinalidade de $B$ é $2^{c}$; isso pode ser comprovado pela bijeção $\psi: A \longrightarrow B$ definida por $\psi\left(H_{C}\right)=H_{C} \circ h$. Portanto, $\operatorname{dim} V=2^{c}$.

Agora, de modo simples, compondo cada função do espaço vetorial $V$ com uma função fortemente Darboux (como, por exemplo, a que foi apresentada no início dessa seção), finalmente podemos demonstrar o resultado principal deste capítulo:

Teorema 2.14. A lineabilidade do conjunto $\mathcal{F} \mathcal{D}(\mathbb{R})$ é $2^{c}$.

Demonstração : Tomemos uma função $f \in \mathcal{F} \mathcal{D}(\mathbb{R})$ arbitrária porém, fixada; seja $D:=\{H \circ f: H \in B\}$ sendo $B$ o conjunto definido no corolário anterior. Afirmamos que o espaço vetorial $W$ gerado por $D$ é um subconjunto de $\mathcal{F} \mathcal{D}(\mathbb{R}) \cup\{0\}$ com dimensão $2^{c}$ :

Tomemos uma função não nula $g \equiv \sum_{j=1}^{n} \beta_{j}\left(H_{j} \circ f\right) \operatorname{com} H_{j} \in B$ e $\beta_{j} \in \mathbb{R}, \forall j$. Sejam $s \in \mathbb{R}$ e $I \subset \mathbb{R}$ um intervalo aberto arbitrários. Precisamos encontrar $l \in I$ tal que $g(l)=s$. Então:

$$
g \equiv \sum_{j=1}^{n} \beta_{j}\left(H_{j} \circ f\right) \equiv\left(\sum_{j=1}^{n} \beta_{j} H_{j}\right) \circ f
$$

Chamando de $G \equiv \sum_{j=1}^{n} \beta_{j} H_{j}$, temos que $G \in V \backslash\{0\}$ e $g \equiv G \circ f$. Como $G$ é sobrejetora, existe $d \in \mathbb{R}$ tal que $G(d)=s$. Agora podemos encontrar $l \in I$ tal que $f(l)=d$ (isto é 
possível pois a $f$ é fortemente Darboux). Logo:

$$
g(l)=(G \circ f)(l)=G(f(l))=G(d)=s
$$

Agora estamos em condições de mostrar que a lineabilidade de $\mathcal{F} \mathcal{D}(\mathbb{R})$ é $2^{c}$, ou seja, que $\lambda(\mathcal{F D}(\mathbb{R}))=2^{c}$ :

Sabemos da teoria dos conjuntos que a cardinalidade do conjunto de todas as funções $g: \mathbb{R} \longrightarrow \mathbb{R}$ é $2^{c}$. Logo, a cardinalidade de $\mathcal{F} \mathcal{D}(\mathbb{R})$ deve ser, no máximo, igual a $2^{c}$. Por outro lado, existe uma bijeção $\phi: B \longrightarrow D$ dada por $\phi(H)=H \circ f$ comprovando que $\operatorname{dim} W=2^{c}$. A demonstração está concluída. 


\section{Conjunto das Raízes de Polinômios Homogêneos Reais}

Vimos, nos capítulos anteriores, que os conjuntos formados pela função identicamente nula e pelas funções patológicas (funções fortemente Darboux e funções diferenciáveis e não monótonas em nenhum intervalo) contêm espaços vetoriais de dimensão infinita, ou seja, são conjuntos lineáveis. Agora, vamos estudar a existência de espaços vetoriais contidos nos conjuntos dos zeros de polinômios. Os autores do texto científico [4] também adotam o termo n-lineável para os conjuntos das raízes dos polinômios homogêneos quando contêm espaços vetoriais de dimensão igual a $n$ e denotam por lineável quando os espaços vetoriais têm dimensão infinita. Citamos aqui os trabalhos [2] e [3] e suas referências que abordam este tema. A última seção desse capítulo está voltada para os polinômios 2-homogêneos $P$ definidos em espaços de Banach reais de dimensão infinita; para tais funções os conjuntos $P^{-1}(0)$ são lineáveis dependendo do tipo de espaço de Banach considerado.

Para apresentarmos exemplos destes conjuntos, necessitamos, primeiramente, de algumas definições e resultados prévios sobre polinômios homogêneos e aplicações multilineares (para mais informações sobre esse assunto ver, por exemplo, [19] e [20]). 


\subsection{Aplicações Multilineares}

O objetivo desta seção é estudar as aplicações multilineares, uma vez que elas estão intrinsicamente ligadas aos polinômios; aqui, é apresentada uma caracterização de continuidade das aplicações multilineares pois todos os polinômios tratados neste capítulo serão assumidos, quando necessário, contínuos. Os resultados estudados nesta seção podem ser encontrados, por exemplo, em [19] e em [20].

Definição 3.1. Sejam $d \in \mathbb{N}$ e $E_{1}, \cdots, E_{d}, F \mathbb{R}$-espaços vetoriais. Uma aplicação $A: E_{1} \times \cdots \times E_{d} \longrightarrow F$ é dita uma aplicação d-linear se as aplicações $x_{i} \longrightarrow A\left(x_{1}, \cdots, x_{i}, \cdots, x_{d}\right)$ forem lineares de $E_{i}$ em $F, \forall i=1,2, \cdots, d$.

Vamos denotar o $\mathbb{R}$-espaço vetorial de todas as aplicações d-lineares de $E_{1} \times \cdots \times E_{d}$ em $F$ por $L_{a}\left(E_{1}, \cdots, E_{d} ; F\right)$. Quando $E=E_{1}=E_{2}=\cdots=E_{d}$, denotamos simplesmente por $L_{a}\left({ }^{d} E ; F\right)$ e, quando $F=\mathbb{R}$, por $L_{a}\left({ }^{d} E\right)$. Por convenção, escrevemos $L_{a}\left({ }^{o} E ; F\right)=F$ e, se $d=1$, então $L_{a}\left({ }^{1} E ; F\right)=L_{a}(E ; F)$.

Sejam $E_{1}, E_{2}, \ldots, E_{d}$ espaços normados sobre $\mathbb{R}$ cujas normas denotamos por $\|\cdot\|_{E_{i}}$, $i=1,2, \cdots, d$, respectivamente. Assim, o produto cartesiano $E_{1} \times \ldots \times E_{d}$ também é um espaço normado quando munido de qualquer umas das seguintes normas:

$$
\begin{aligned}
\|x\|_{\infty} & =\max _{1 \leq i \leq m}\left\|x_{i}\right\|_{E_{i}} \\
\|x\|_{p} & =\left(\left\|x_{1}\right\|_{E_{1}}^{p}+\cdots+\left\|x_{d}\right\|_{E_{d}}^{p}\right)^{\frac{1}{p}},(1 \leq p<\infty),
\end{aligned}
$$

com $x=\left(x_{1}, \ldots, x_{d}\right) \in E_{1} \times \ldots \times E_{d}$. Estas normas são equivalentes e cada uma delas induz a topologia produto em $E_{1} \times \ldots \times E_{d}$. Trabalharemos com a norma $\|\cdot\|_{\infty}$ que, por simplificação, será denotada por $\|\cdot\|$. Quando for necessário o uso de outra norma, ela será escrita na forma que foi apresentada acima.

O próximo teorema nos apresenta algumas equivalências sobre a continuidade de uma aplicação multilinear:

Teorema 3.2. Seja $d \in \mathbb{N}$. Se $E_{1}, E_{2}, \ldots, E_{d}$ e $F$ são espaços normados sobre $\mathbb{R} e$ $A: E_{1} \times \ldots \times E_{d} \longrightarrow F$ uma aplicação multilinear, então são equivalentes:

a) A é contínua; 
b) A é contínua na origem;

c) Existe uma constante $M>0$ tal que $\left\|A\left(x_{1}, \ldots, x_{d}\right)\right\| \leq M\left\|x_{1}\right\| \cdots\left\|x_{d}\right\|$ para qualquer $\left(x_{1}, \cdots, x_{d}\right) \in E_{1} \times \ldots \times E_{d}$.

Demonstração : a) $\Rightarrow$ b) Evidente.

b) $\Rightarrow$ c) Para qualquer $x=\left(x_{1}, \ldots, x_{d}\right) \in E_{1} \times \ldots \times E_{d}$, se $x_{1}=0$, então $A\left(0, x_{2}, \ldots, x_{d}\right)=A\left(0+0, x_{2}, \ldots, x_{d}\right) \Longrightarrow A\left(0, x_{2}, \ldots, x_{d}\right)=0$. De modo análogo, temos que, se $x_{i}=0$, para algum $i=2, \ldots, d$, então $A\left(x_{1}, \ldots, x_{i}, \ldots, x_{d}\right)=0$ e o resultado fica satisfeito $\forall M \in \mathbb{R}$.

Suponhamos, agora, que, para $x=\left(x_{1}, \ldots, x_{d}\right) \in E_{1} \times \ldots \times E_{d}, x_{i} \neq 0, \forall i=1,2, \ldots, d$; por hipótese, dado $\epsilon>0$, existe $\delta>0$ tal que $\forall x=\left(x_{1}, \ldots, x_{d}\right) \in E_{1} \times \ldots \times E_{d}$, $\|x\| \leq \delta \Longrightarrow\|A(x)\| \leq \epsilon$. Desta forma, $\forall x=\left(x_{i}, x_{2}, \ldots, x_{d}\right) \in E_{1} \times \ldots \times E_{d}$, tomando-se $y \in E_{1} \times \ldots \times E_{d}$ tal que $y=\left(k \cdot \delta \cdot \frac{x_{1}}{\left\|x_{1}\right\|}, \ldots, k \cdot \delta \cdot \frac{x_{d}}{\left\|x_{d}\right\|}\right)$ para algum escalar $k$ com $0<|k| \leq 1$, temos que

$\|y\|=|k| \cdot \delta \leq \delta$. Com isso, $\|A(y)\| \leq \epsilon \Longrightarrow\left\|A\left(x_{1}, \ldots, x_{d}\right)\right\| \leq \frac{\epsilon}{|k|^{d} \cdot \delta^{d}} \cdot\left\|x_{1}\right\| \ldots\left\|x_{d}\right\|$. Tomando-se $M=\frac{\epsilon}{|k|^{d} \cdot \delta^{d}}$, obtemos a desigualdade proposta em c).

c) $\Rightarrow$ a) Sejam $r B_{E_{1} \times \cdots \times E_{d}}$ a bola aberta de $E_{1} \times \cdots \times E_{d}$ de raio $r>0 \mathrm{e}$ $a=\left(a_{1}, \ldots, a_{d}\right) \in r B_{E_{1} \times \cdots \times E_{d}}$. Então, $\forall x=\left(x_{1}, \ldots, x_{d}\right) \in r B_{E_{1} \times \cdots \times E_{d}}$, temos que $\left\|x_{i}\right\| \leq\|x\|<r$ e, como $\left\|a_{i}\right\| \leq\|a\|<r, i=1, \ldots, d$, segue:

$$
\begin{aligned}
A(x)-A(a)= & A\left(x_{1}-a_{1}, x_{2}, \ldots, x_{d}\right)+A\left(a_{1}, x_{2}-a_{2}, \ldots, x_{d}\right) \\
& +\cdots+A\left(a_{1}, \ldots, a_{d-1}, x_{d}-a_{d}\right) \Longrightarrow \\
\Longrightarrow\|A(x)-A(a)\| \leq & \left\|A\left(x_{1}-a_{1}, x_{2}, \ldots, x_{d}\right)\right\|+\left\|A\left(a_{1}, x_{2}-a_{2}, \ldots, x_{d}\right)\right\| \\
& +\ldots+\left\|A\left(a_{1}, \ldots, a_{d-1}, x_{d}-a_{d}\right)\right\| \leq \\
\leq & M\left\|x_{1}-a_{1}\right\| \cdot\left\|x_{2}\right\| \ldots\left\|x_{d}\right\|+M\left\|a_{1}\right\| \cdot\left\|x_{2}-a_{2}\right\| \ldots\left\|x_{d}\right\| \\
& +\cdots+M\left\|a_{1}\right\| \ldots\left\|a_{d-1}\right\| \cdot\left\|x_{d}-a_{d}\right\| \leq
\end{aligned}
$$




$$
\begin{aligned}
& \leq M \cdot r^{d-1}\left(\left\|x_{1}-a_{1}\right\|+\ldots+\left\|x_{d}-a_{d}\right\|\right) \leq \\
& \leq M \cdot r^{d-1} \cdot\|x-a\|_{1} .
\end{aligned}
$$

Como $x$ foi tomado arbitrariamente em $r B_{E_{1} \times \cdots \times E_{d}}$, temos que $A$ é uniformemente contínua em $r B_{E_{1} \times \cdots \times E_{d}}, \forall r>0$. Logo, $A$ é contínua.

Observação: O teorema anterior mostrou que toda aplicação d-linear é uniformemente contínua sobre conjuntos limitados; no entanto, exceto para o caso linear, nenhuma aplicação d-linear $A$ não nula é uniformemente contínua, ou seja, deve ocorrer:

Para $\delta>0$, existe $\epsilon>0$ tal que, para $x=\left(x_{1}, \ldots, x_{d}\right) \in E_{1} \times \ldots E_{d}$ com $\|x\|<\delta$, tenhamos $\|A(x)\| \geq \epsilon$.

De fato, seja $A$ uma aplicação d-linear não nula, $d \geq 2$. Então existe $\left(x_{1}, x_{2}, \ldots, x_{d}\right)$ tal que $\left\|A\left(x_{1}, x_{2}, \ldots, x_{d}\right)\right\|>r>0$. Cada $x_{i}$ é, então, não nulo. Se $\epsilon=r$, para qualquer $\delta>0$, podemos escolher $\lambda \in \mathbb{R}$ tal que $0<|\lambda|<\frac{\delta}{\left\|x_{1}\right\|}$, ou seja, $\left\|\lambda x_{1}\right\|<\delta$. Com isso, $\left\|\left(x_{1}+\lambda x_{1}, \frac{x_{2}}{\lambda}, \ldots, x_{d}\right)-\left(x_{1}, \frac{x_{2}}{\lambda}, \ldots, x_{d}\right)\right\|=\left\|\lambda x_{1}\right\|<\delta$.

Mas

$$
\begin{aligned}
\left\|A\left(x_{1}+\lambda x_{1}, \frac{x_{2}}{\lambda}, \ldots, x_{d}\right)-A\left(x_{1}, \frac{x_{2}}{\lambda}, \ldots, x_{d}\right)\right\| & =\left\|A\left(\lambda x_{1}, \frac{x_{2}}{\lambda}, \ldots, x_{d}\right)\right\| \\
& =\left\|A\left(x_{1}, x_{2}, \ldots, x_{d}\right)\right\|>r .
\end{aligned}
$$

Logo, $A$ não é uniformemente contínua.

É claro que se uma aplicação multilinear é contínua, então ela é contínua em cada variável separadamente; porém, a recíproca nem sempre é verdadeira como mostra o seguinte exemplo:

Exemplo 3.3. Seja $E=\mathfrak{C}_{L_{1}}([0 ; 1])$, o espaço vetorial das funções contínuas de $[0 ; 1]$ em $\mathbb{R}$ munido da norma $\|x\|=\int_{0}^{1}|x(t)| d t . \quad A$ aplicação $B \in L_{a}\left({ }^{2} E\right)$ definida por $B(x, y)=\int_{0}^{1} x(t) y(t) d t$ é separadamente contínua, mas não é contínua. 
Vamos ver primeiro que $B$ é separadamente contínua. Para cada $x$ de $E$ fixo, temos $|B(x, y)| \leq \int_{0}^{1}|x(t)| \cdot|y(t)| d t \leq \int_{0}^{1}\|x\|_{\infty}|y(t)| d t=\|x\|_{\infty} \int_{0}^{1}|y(t)| d t=\|x\|_{\infty}\|y\|$, ou seja, é contínua na segunda variável. Da mesma forma mostramos que $B$ é contínua na primeira variável. Assim, $B$ é contínua separadamente. Para ver que $B$ não é contínua, consideremos a seqüência

$$
x_{n}(t)= \begin{cases}n-n^{3} t & , \text { se } 0 \leq t \leq \frac{1}{n^{2}} \\ 0 & , \text { se } \frac{1}{n^{2}} \leq t \leq 1\end{cases}
$$

Vemos, então, que

$$
\left\|x_{n}\right\|=\int_{0}^{1}\left|n-n^{3} t\right| d t=\int_{0}^{\frac{1}{n^{2}}}\left(n-n^{3} t\right) d t=\frac{1}{2 n} .
$$

Portanto, $\left(x_{n}, x_{n}\right) \longrightarrow 0$. Mas

$$
B\left(x_{n}, x_{n}\right)=\int_{0}^{\frac{1}{n^{2}}}\left(n-n^{3} t\right)^{2} d t=\frac{1}{3},
$$

que não converge para zero.

Porém, se cada espaço $E_{i}$ definido no teorema 3.2 é de Banach, temos, então, uma caracterização da continuidade de uma aplicação multilinear com relação a continuidade em cada uma de suas variáveis:

Teorema 3.4. Sejam $E_{1}, E_{2}, \ldots, E_{d}$ espaços de Banach e $F$ um espaço normado. Então, $A \in L_{a}\left(E_{1}, \ldots, E_{d} ; F\right)$ é contínua se, e somente se, é contínua em cada variável.

Demonstração : Ver, por exemplo, em [19], pg. 12 a 14.

Corolário 3.5. Uma multilinear que está definida em um produto de espaços de dimensão finita é sempre contínua. 
Demonstração : Basta observar que, nesse caso, cada espaço é de Banach e toda linear definida em espaço de dimensão finita é contínua.

Na seção seguinte, vamos trabalhar com polinômios homogêneos definidos em espaços de dimensão finita $\left(E_{i}=\mathbb{R}^{m}, \forall i, m \in \mathbb{N}\right)$ enquanto que polinômios 2-homogêneos definidos em espaços de Banach de dimensão infinita serão tratados na seção 3.4; para esses casos, vamos assumir que os polinômios sejam contínuos. Portanto, estaremos sempre trabalhando com aplicações multilineares contínuas.

Denotamos por $L\left(E_{1}, \cdots, E_{d} ; F\right)$ o subespaço das transformações multilineares contínuas de $L_{a}\left(E_{1}, \cdots, E_{d} ; F\right)$. Da mesma forma, quando $E=E_{1}=\cdots=E_{d}$, escreve$\operatorname{mos} L\left({ }^{d} E ; F\right)$ e, quando $F=\mathbb{R}$, denotamos $L\left(E_{1}, \cdots, E_{d} ; F\right)$ por $L\left(E_{1}, \cdots, E_{d}\right)$. Para $d=1$, escrevemos $L(E ; F)$.

Como estamos interessados em polinômios, restringimo-nos ao caso $E=E_{1}=\cdots=$ $E_{d}$. Como base para esse estudo, usamos aplicações multilineares simétricas cuja definição é a seguinte:

Definição 3.6. Sejam E e $F \mathbb{R}$-espaços vetoriais. Uma aplicação d-linear $A: E^{d} \longrightarrow F$ é dita simétrica se $\forall x_{1}, \cdots, x_{d} \in E, A\left(x_{1}, \cdots, x_{d}\right)=A\left(x_{\sigma(1)}, \cdots, x_{\sigma(d)}\right)$, $\forall \sigma \in S_{d}$ sendo $S_{d}$ o grupo de permutações de d elementos.

Denotamos por $L_{a s}\left({ }^{d} E ; F\right)$ o espaço das transformações d-lineares simétricas de $E^{d}$ em $F$. Para $E$ e $F$ normados, denotamos por $L_{s}\left({ }^{d} E ; F\right)$ o espaço das aplicações d-lineares simétricas contínuas de $E^{d}$ em $F$.

Com o objetivo de simplificar a notação, para qualquer aplicação multilinear $A \in L_{a}\left({ }^{d} E ; F\right)$, vamos usar a seguinte convenção:

Definição 3.7. Sejam $E$ e $F$ espaços vetoriais sobre $\mathbb{R}$. Sejam $A \in L_{a}\left({ }^{d} E ; F\right) e$ $k \in \mathbb{N}, k \leq d . \forall x_{1}, \ldots, x_{k} \in E$ e $\forall n_{1}, \cdots, n_{k} \in \mathbb{N}$ com $n_{1}+\cdots+n_{k}=d$, definimos

$$
A x_{1}^{n_{1}} \cdots x_{k}^{n_{k}}=\left\{\begin{array}{cl}
A(\overbrace{x_{1}, \cdots, x_{1}}^{n_{1} \text { vezes }}, \overbrace{x_{2}, \cdots, x_{2}}^{n_{2} \text { vezes }}, \cdots, \overbrace{x_{k}, \cdots, x_{k}}^{n_{k} \text { vezes }} & \text { se } d \geq 1 \\
A & \text { se } d=0
\end{array}\right.
$$


Com essa notação, podemos enunciar o teorema que nos mostra que uma aplicação multilinear simétrica depende apenas de seus valores na diagonal do espaço $E^{d}$; ela será útil no estudo dos polinômios.

Teorema 3.8. (Fórmula de Polarização): Sejam E e F R-espaços vetoriais e $A \in L_{a s}\left({ }^{d} E ; F\right)$. Então, $\forall x_{1}, \cdots, x_{d} \in E$, temos:

$$
A\left(x_{1}, \cdots, x_{d}\right)=\frac{1}{d ! 2^{d}} \sum_{\substack{\epsilon_{i}= \pm 1 \\ 1 \leq i \leq d}} \epsilon_{1} \cdots \epsilon_{d} \cdot A\left(\epsilon_{1} x_{1}+\cdots+\epsilon_{d} x_{d}\right)^{d} .
$$

Demonstração : ver, por exemplo, em [19], pg. 20 e 21.

A partir das transformações multilineares definimos polinômios homogêneos.

\subsection{Polinômios Homogêneos}

O objetivo desta seção é apresentar um teorema referente a uma caracterização de continuidade dos polinômios homogêneos definidos em espaços de Banach $E$; tal caracterização será usada na seção 3.4 enquanto que, na seção 3.3, estaremos trabalhando com polinômios definidos em $E=\mathbb{R}^{m}$.

Definições 3.9. Sejam E e F R-espaços vetoriais.

a) Dizemos que uma função $P: E \longrightarrow F$ é um polinômio homogêneo de grau d (ou um polinômio d-homogêneo) se existe uma aplicação d-linear $A: E^{d} \longrightarrow F$ tal que $P(x)=A x^{d}$ para todo $x \in E$. Dizemos que $P$ é o polinômio d-homogêneo associado a A ou que A é uma transformação d-linear associada a $P$.

b) Dizemos que uma função $P: E \longrightarrow F$ é um polinômio de grau d se $P$ pode ser representado como

$$
P=P_{0}+P_{1}+\ldots+P_{d}
$$

sendo $P_{j} \in P_{a}\left({ }^{j} E ; F\right)$ para cada $j=0,1, \ldots, d$, com $P_{d} \neq 0$. 
Denotamos por $P_{a}\left({ }^{d} E ; F\right)$ o espaço vetorial de todos os polinômios d-homogêneos de $E$ em $F$. Quando $F=\mathbb{R}$, escrevemos apenas $P_{a}\left({ }^{d} E\right)$. Para $d=0, P(x)=A x^{0}=A \in F$ sendo uma função constante.

Com o próximo teorema, vamos restringir as multilineares associadas a um polinômio homogêneo:

Teorema 3.10. Sejam $E$ e $F \mathbb{R}$-espaços vetoriais e $d \in \mathbb{N}$. Se $P \in P_{a}\left({ }^{d} E ; F\right)$, então existe uma única aplicação d-linear simétrica $A_{s} \in L_{a s}\left({ }^{d} E ; F\right)$ tal que $P(x)=A_{s} x^{d}$ para todo $x \in E$.

Demonstração : Basta tomarmos a aplicação d-linear simétrica $A_{s}$ definida por:

$$
A_{s}\left(x_{1}, \cdots, x_{d}\right)=\frac{1}{d !} \sum_{\sigma \in S_{d}} A\left(x_{\sigma(1)}, \cdots, x_{\sigma(d)}\right)
$$

Desta forma, temos $A_{s}\left(x^{d}\right)=P(x)$ e fica, assim, mostrada a sua existência. Sejam, agora, $A_{1}$ e $A_{2}$ duas aplicações d-lineares simétricas associadas a $P$; então, pela fórmula de polarização, é imediato que $A_{1}\left(x_{1}, \ldots, x_{d}\right)=A_{2}\left(x_{1}, \ldots, x_{d}\right), \forall\left(x_{1}, \ldots, x_{d}\right) \in E_{1} \times \ldots \times E_{d}$. Isto mostra a unicidade da aplicação simétrica associada a $P$.

Com isso, podemos estabelecer uma importante relação entre os espaços $L_{a s}\left({ }^{d} E ; F\right) \mathrm{e}$ $P_{a}\left({ }^{d} E ; F\right)$ como mostra a seguinte proposição:

Proposição 3.11. A função $A \mapsto P$ é um isomorfismo de espaços vetoriais entre $L_{a s}\left({ }^{d} E ; F\right)$ e $P_{a}\left({ }^{d} E ; F\right)$, para cada $d \in \mathbb{N}$.

Demonstração : A bijeção é conseqüência imediata do teorema anterior. Para mostrar que a função é linear, tomemos $A_{1}, A_{2} \in L_{a s}\left({ }^{d} E ; F\right), \alpha \in \mathbb{R}$ e $x \in E$. Tomando-se os polinômios $P_{1}, P_{2} \in P_{a}\left({ }^{d} E ; F\right)$ associados a $A_{1}$ e $A_{2}$, respectivamente, temos:

$$
\left(\alpha P_{1}+P_{2}\right)(x)=\left(\alpha A_{1}+A_{2}\right) x^{d}=\alpha A_{1} x^{d}+A_{2} x^{d}=\alpha P_{1}(x)+P_{2}(x),
$$


O seguinte teorema fornece critérios para a continuidade de um polinômio $d$-homogêneo.

Teorema 3.12. Sejam $E$ e $F$ espaços normados sobre $\mathbb{R}$. Sejam $d \in \mathbb{N}, P \in P_{a}\left({ }^{d} E ; F\right)$ e $A \in L_{a s}\left({ }^{d} E ; F\right)$ associada a $P$. Então, as seguintes afirmações são equivalentes:

a) $A \in L_{s}\left({ }^{d} E ; F\right)$;

b) $P \in P\left({ }^{d} E ; F\right)$;

c) P é contínuo na origem;

d) Existe uma constante $M>0$, tal que $\|P(x)\| \leq M\|x\|^{d}$ para qualquer $x \in E$.

Demonstração : Para $d=0$, as equivalências são triviais uma vez que o polinômio $P$ é constante. Suponhamos $d \geq 1$ :

a) $\Rightarrow$ b) Evidente.

b) $\Rightarrow$ c) Evidente.

c) $\Rightarrow$ d) Por hipótese, dado $\epsilon>0$, existe $\delta>0$ tal que, $\forall x \in E$ com $\|x\| \leq \delta$, $\|P(x)\| \leq \epsilon$. Para qualquer $x \in E$ não nulo, tomando-se $y=k \cdot \delta \cdot \frac{x}{\|x\|}$ para algum escalar $k$ com $0<|k| \leq 1$, temos que ||$y||=|k| \cdot \delta \leq \delta$. Desta forma,

$$
\begin{gathered}
\|P(y)\| \leq \epsilon \Longrightarrow\left\|A\left(k \cdot \delta \cdot \frac{x}{\|x\|}\right)^{d}\right\| \leq \epsilon \Longrightarrow\left\|A\left(x^{d}\right)\right\| \leq \frac{\epsilon}{k^{d} \cdot \delta^{d}} \cdot\|x\|^{d}=M \cdot\|x\|^{d} \Longrightarrow \\
\Longrightarrow\|P(x)\| \leq M \cdot\|x\|^{d}, \forall x \in E \text { não nulo. }
\end{gathered}
$$

Como $P(0)=0$, obtemos a desigualdade proposta em d).

d) $\Rightarrow$ a) Sejam $x_{1}, \ldots, x_{d} \in E$ não nulos arbitrários. Dado $\delta>0$, seja $y=\left(\delta \cdot \frac{x_{1}}{\left\|x_{1}\right\|}, \ldots \delta \cdot \frac{x_{d}}{\left\|x_{d}\right\|}\right) \in E^{d}$. Por hipótese, seja $M>0$ tal que $\|P(x)\| \leq M \cdot\|x\|^{d}$, qualquer $x \in E$ e, usando a fórmula de polarização, obtemos: 


$$
\begin{aligned}
& \|A(y)\| \leq \frac{1}{d ! 2^{d}} \sum_{\substack{\epsilon_{i}= \pm 1 \\
1 \leq i \leq d}}\left|\epsilon_{1}\right| \cdots\left|\epsilon_{d}\right|\left\|A\left(\epsilon_{1} \cdot \delta \frac{x_{1}}{\left\|x_{1}\right\|}+\cdots \epsilon_{d} \cdot \delta \frac{x_{d}}{\left\|x_{d}\right\|}\right)^{d}\right\|= \\
& =\frac{1}{d ! 2^{d}} \sum_{\substack{\epsilon_{i}= \pm 1 \\
1 \leq i \leq d}}\left\|P\left(\epsilon_{1} \cdot \delta \frac{x_{1}}{\left\|x_{1}\right\|}+\cdots+\epsilon_{d} \cdot \delta \frac{x_{d}}{\left\|x_{d}\right\|}\right)\right\| \leq \\
& \left.\leq \frac{1}{d ! 2^{d}} \sum_{\substack{\epsilon_{i}= \pm 1 \\
1 \leq i \leq d}} M \cdot \| \epsilon_{1} \cdot \delta \frac{x_{1}}{\left\|x_{1}\right\|}+\cdots+\epsilon_{d} \cdot \delta \frac{x_{d}}{\left\|x_{d}\right\|}\right) \|^{d} \leq \\
& \leq \frac{1}{d ! 2^{d}} \sum_{\substack{\epsilon_{i}= \pm 1 \\
1 \leq i \leq d}} M\left(\left\|\epsilon_{1} \cdot \delta \frac{x_{1}}{\left\|x_{1}\right\|}\right\|+\cdots+\left\|\epsilon_{d} \cdot \delta \frac{x_{d}}{\left\|x_{d}\right\|}\right\|\right)^{d}= \\
& =\frac{1}{d ! 2^{d}} \sum_{\substack{c_{i}= \pm 1 \\
1 \leq i \leq d}} M \cdot \delta^{d} \cdot d^{d}=\frac{1}{d ! 2^{d}} 2^{d} \cdot M \cdot \delta^{d} \cdot d^{d}=\frac{M \cdot \delta^{d} \cdot d^{d}}{d !} \Longrightarrow \\
& \Longrightarrow \delta^{d} \cdot \frac{\left\|A\left(x_{1}, \ldots, x_{d}\right)\right\|}{\left\|x_{1}\right\| \ldots\left\|x_{d}\right\|} \leq \frac{M \cdot \delta^{d} \cdot d^{d}}{d !} \Longrightarrow\left\|A\left(x_{1}, \ldots, x_{d}\right)\right\| \leq \frac{M \cdot d^{d}}{d !} \cdot\left\|x_{1}\right\| \ldots\left\|x_{d}\right\| .
\end{aligned}
$$

Como essa desigualdade vale também se $x_{i}=0$ para algum $i=1, \ldots, d$, pelo teorema 3.2 , a aplicação simétrica $A$ é contínua.

O teorema anterior mostrou que, em qualquer conjunto limitado, um polinômio homogêneo é contínuo se, e somente se, é limitado.

A proposição seguinte mostra que os polinômios contínuos são uniformemente contínuos em conjuntos limitados:

Proposição 3.13. Sejam E, $F$ espaços normados sobre $\mathbb{R}$ e $P \in P\left({ }^{d} E ; F\right)$. Então $P$ é uniformemente contínuo sobre os limitados. 
Demonstração : Seja $A$ uma aplicação d-linear associada a $P$. Para qualquer $r>0$, seja $r B_{E}$ a bola aberta de $E$ de raio $r$. Tomando-se $x \in r B_{E}$ arbitrariamente, temos que $\|x\|<r$ e como $A$ é contínua, pelo teorema 3.2, existe uma constante $M>0$ tal que $\|P(x)\|=\left\|A\left(x^{d}\right)\right\| \leq M \cdot\|x\|^{d} \leq M \cdot r^{d-1} \cdot\|x\|$. Chamando de $M_{1}=M \cdot r^{d-1}$, temos $\|P(x)\| \leq M_{1} \cdot\|x\|$ e, com isso, $P$ é uniformemente contínuo em $r B_{E}, \forall r>0$. Como cada subconjunto limitado de $E$ está contido em $r B_{E}$ para algum $r>0$, temos que $P$ é uniformemente contínuo em cada limitado de $E$.

As aplicações multilineares com que vamos trabalhar são contínuas; desta forma, podemos concluir que os polinômios associados a elas também o são.

Denotamos por $P\left({ }^{d} E ; F\right)$ o subespaço vetorial de $P_{a}\left({ }^{d} E ; F\right)$ formado pelos polinômios d-homogêneos contínuos de $E$ em $F$. Quando $F=\mathbb{R}$, usamos a notação $P\left({ }^{d} E\right)$.

\subsection{Polinômios Homogêneos Definidos em $\mathbb{R}^{m}$}

Nesta seção, estamos interessados em estudar os conjuntos das raízes de polinômios reais homogêneos quanto ao fato deles conterem espaços vetoriais, ou seja, serem lineáveis; vamos, aqui, considerar $E=\mathbb{R}^{m}$. Lembramos que, para qualquer funcional linear definido em $\mathbb{R}^{m}$, o núcleo é um hiperplano de $\mathbb{R}^{m}$. Então, para qualquer funcional $f \in\left(\mathbb{R}^{m}\right)^{\prime}$, temos que $f^{-1}(0)$ é $(m-1)$-lineável.

Para os polinômios 2-homogêneos, usamos algumas definições e resultados da álgebra linear relacionados às aplicações bilineares.

Definição 3.14. Sejam $E$ um $\mathbb{R}$-espaço vetorial de dimensão $n \geq 1$ e $B=\left\{e_{1}, e_{2}, \cdots, e_{n}\right\}$ uma base de $E$. Para cada $A \in L\left({ }^{2} E\right)$, definimos a matriz de $A$ em relação à base ordenada $B$ como $[A]_{B}=\left(A\left(e_{i}, e_{j}\right)\right)_{i, j}$ pertencente à $M_{n}(\mathbb{R})$, o espaço das matrizes $n \times n$ sobre $\mathbb{R}$.

Em particular, existe um isomorfismo entre $L\left({ }^{2} E\right)$ e $M_{n}(\mathbb{R})$. De fato, basta definirmos a transformação $T: L\left({ }^{2} E\right) \longrightarrow M_{n}(\mathbb{R})$ por $T(A)=[A]_{B}$. 
O próximo resultado relaciona aplicações bilineares simétricas e matrizes diagonais:

Teorema 3.15. Seja $E$ um $\mathbb{R}$-espaço vetorial de dimensão $n \geq 1$. Se $A \in L_{s}\left({ }^{2} E\right)$, então existe uma base $B$ de $E$ tal que $[A]_{B}$ é uma matriz diagonal.

Demonstração : Ver, por exemplo, [10], pg. 229.

Podemos, então, determinar a dimensão de um espaço vetorial formado pelas raízes dos polinômios reais 2-homogêneos definidos em $\mathbb{R}^{m}$ sabendo-se apenas as quantidades de auto-valores da matriz simétrica $A$ associada a $P$ :

Proposição 3.16. Seja $P \in P\left({ }^{2} \mathbb{R}^{m}\right)$. Então $P^{-1}(0)$ é r-lineável com $r=\min \{p, n\}+z$ sendo $p, n$ e $z$ as quantidades de auto-valores, respectivamente positivos, negativos e nulos da matriz simétrica associada a $P$.

Demonstração : Seja $\left[A_{s}\right] \in M_{m}(\mathbb{R})$ a matriz simétrica associada a $P$. Então, $\left[A_{s}\right]$ é diagonalizável; seja $B=\left\{e_{1}, e_{2}, \cdots, e_{m}\right\}$ a base ordenada de $\mathbb{R}^{m}$ tal que a matriz $\left[A_{s}\right]_{B}$ é diagonal com $\left(\lambda_{j}\right)_{j=1}^{m}$ os seus auto-valores colocados da seguinte maneira:

a) Os auto-valores positivos $\left(\lambda_{j}\right)_{j=1}^{p}$ são os elementos $a_{j j}=\lambda_{j}$ da matriz $\left[A_{s}\right]_{B}$ ( $p$ auto-valores positivos).

b) Os auto-valores negativos $\left(-\lambda_{j}\right)_{j=p+1}^{p+n}, \lambda_{j}>0, j=p+1, p+2, \cdots, p+n$, são os elementos $a_{j j}=-\lambda_{j}$ da matriz $\left[A_{s}\right]_{B}$ ( $n$ auto-valores negativos).

c) Os auto-valores nulos são os elementos $a_{j j}$ para $j=p+n+1, p+n+2, \cdots, m$ ( $z$ auto-valores nulos).

Como $P(x)=A_{s}(x, x), \forall x \in \mathbb{R}^{m}$, temos:

$$
P\left(e_{j}\right)=A_{s}\left(e_{j}, e_{j}\right)= \begin{cases}\lambda_{j} & \forall j=1,2, \cdots, p \\ -\lambda_{j} & \forall j=p+1, \cdots, p+n \\ 0 & \forall j=p+n+1, \cdots, m\end{cases}
$$


Suponhamos que $p \leq n$ (para $p \geq n$, a demonstração é análoga). Então, para $j=1, \ldots, p$, segue:

$$
\begin{gathered}
P\left(e_{j}+\sqrt{\frac{\lambda_{j}}{\lambda_{p+j}}} e_{p+j}\right)=A_{s}\left(e_{j}+\sqrt{\frac{\lambda_{j}}{\lambda_{p+j}}} e_{p+j}, e_{j}+\sqrt{\frac{\lambda_{j}}{\lambda_{p+j}}} e_{p+j}\right)= \\
=A_{s}\left(e_{j}, e_{j}\right)+A_{s}\left(e_{j}, \sqrt{\frac{\lambda_{j}}{\lambda_{p+j}}} e_{p+j}\right)+A_{s}\left(\sqrt{\frac{\lambda_{j}}{\lambda_{p+j}}} e_{p+j}, e_{j}\right)+ \\
+A_{s}\left(\sqrt{\frac{\lambda_{j}}{\lambda_{p+j}}} e_{p+j}, \sqrt{\frac{\lambda_{j}}{\lambda_{p+j}}} e_{p+j}\right)= \\
=\lambda_{j}+0+0+\left(\sqrt{\frac{\lambda_{j}}{\lambda_{p+j}}}\right)^{2}\left(-\lambda_{p+j}\right)=0
\end{gathered}
$$

Com isso, todos os vetores da forma $\left(e_{j}+\sqrt{\frac{\lambda_{j}}{\lambda_{p+j}}} e_{p+j}\right) \in P^{-1}(0)$, para todo $j=1,2, \cdots, p$.

Além desses vetores, $e_{i} \in P^{-1}(0), \forall i=p+n+1, \cdots, m$. Desta forma, o espaço vetorial gerado pelo conjunto

$$
\left\{e_{1}+\sqrt{\frac{\lambda_{1}}{\lambda_{p+1}}} e_{p+1}, e_{2}+\sqrt{\frac{\lambda_{2}}{\lambda_{p+2}}} e_{p+2}, \cdots, e_{p}+\sqrt{\frac{\lambda_{p}}{\lambda_{2 p}}} e_{2 p}, e_{p+n+1}, \cdots, e_{m}\right\}
$$

está contido em $P^{-1}(0)$ e tem dimensão igual a $r=p+z$. Portanto, $P^{-1}(0)$ é r-lineável.

No que segue, vamos exibir exemplos de valores de $r$ dado na proposição 3.16 :

Exemplos 3.17. Para todos os exemplos, vamos considerar que $P \in P\left({ }^{2} \mathbb{R}^{m}\right)$ e $x=\left(x_{1}, \cdots, x_{m}\right) \in \mathbb{R}^{m}$ arbitrário.

1) Seja $P(x)=\sum_{j=1}^{m} x_{j}^{2}$. Então, a aplicação bilinear simétrica tem como matriz diagonal a própria matriz identidade de ordem $m$. Com isso, $p=m$ e $n=z=0$. Logo, $r=0$ 
2) Seja $P(x)=\sum_{j=1}^{m}(-1)^{j} x_{j}^{2}$. Procedendo analogamente ao que foi feito na demonstração da proposição 3.16, concluímos que $r=\left[\frac{m}{2}\right]$ sendo $[a]$ o maior inteiro, menor ou igual a $a, \forall a \in \mathbb{R}$.

3) Seja, $\forall m \geq 3, P(x)=x_{1}^{2}+2 x_{2} x_{3}$. Então, $\forall y=\left(y_{1}, y_{2}, \cdots, y_{m}\right) \in \mathbb{R}^{m}$, a aplicação bilinear simétrica associada a $P$ é dada por $A_{s}(x, y)=x_{1} y_{1}+x_{2} y_{3}+x_{3} y_{2}$; da definição 3.14, considerando-se a base canônica de $\mathbb{R}^{m}$, temos:

$$
\Longrightarrow \quad\left[A_{s}\right]_{c a n}=\left(\begin{array}{ccccc}
1 & 0 & 0 & \ldots & 0 \\
0 & 0 & 1 & \ldots & 0 \\
0 & 1 & 0 & \ldots & 0 \\
\vdots & & & & \\
0 & 0 & 0 & \ldots & 0
\end{array}\right)_{m \times m} .
$$

Logo, tomando-se uma base $B=\left\{v_{1}, v_{2}, \cdots, v_{m}\right\}$ formada por auto-vetores, a matriz diagonal é:

$$
\left[A_{s}\right]_{B}=\left(\begin{array}{ccccc}
1 & 0 & 0 & \cdots & 0 \\
0 & 1 & 0 & \cdots & 0 \\
0 & 0 & -1 & \cdots & 0 \\
\vdots & & & & \\
0 & 0 & 0 & \cdots & 0
\end{array}\right)_{m \times m}
$$

Neste caso, $\quad p=2, n=1$ e $z=m-3 \Longrightarrow r=1+m-3 \Longrightarrow r=m-2$.

Podemos ver, com isso, que o conjunto $\left\{v_{1}+v_{3}, v_{4}, v_{5}, \cdots, v_{m}\right\}$ gera um espaço vetorial de dimensão $m-2$ contido em $P^{-1}(0)$. Portanto, $P^{-1}(0)$ é $(m-2)$ lineável. Para verificação, basta calcular $P$ nos elementos deste conjunto gerador:

$$
\begin{gathered}
P\left(v_{1}+v_{3}\right)=A_{s}\left(v_{1}+v_{3}, v_{1}+v_{3}\right)=A_{s}\left(v_{1}, v_{1}\right)+A_{s}\left(v_{1}, v_{3}\right)+A_{s}\left(v_{3}, v_{1}\right)+A_{s}\left(v_{3}, v_{3}\right) \Longrightarrow \\
\Longrightarrow P\left(v_{1}+v_{3}\right)=1+0+0-1=0 .
\end{gathered}
$$

Além disso, $P\left(v_{i}\right)=A_{s}\left(v_{i}, v_{i}\right)=0, \forall i=4,5, \cdots, m$ 
Para polinômios $d$-homogêneos com $d$ ímpar $\geq 3$, vamos usar o conceito de polinômios simétricos, a saber:

Definição 3.18. Seja $P \in P\left({ }^{d} \mathbb{R}^{m}\right)$; dizemos que $P$ é um polinômio d-homogêneo simétrico se $P\left(x_{1}, \cdots, x_{m}\right)=P\left(x_{\pi(1)}, \cdots, x_{\pi(m)}\right)$ para toda permutação $\pi$ de $S_{m}$.

Observamos que o polinômio 2-homogêneo definido no exemplo 3.17 item 1) é simétrico.

Como a soma, a diferença e o produto de dois polinômios homogêneos simétricos (não necessariamente de mesmo grau) definidos em $\mathbb{R}^{m}$ apresentam a propriedade de serem invariantes com relação a qualquer permutação de $S_{m}$, podemos, naturalmente, estender o conceito de simetria para polinômios como mostra a seguinte definição:

Definição 3.19. Seja $P: \mathbb{R}^{m} \longrightarrow \mathbb{R}$ um polinômio de grau d; dizemos que $P$ é um polinômio simétrico se $P\left(x_{1}, \cdots, x_{m}\right)=P\left(x_{\pi(1)}, \cdots, x_{\pi(m)}\right)$ para toda permutação $\pi$ de $S_{m}$.

Apesar da semelhança de nomenclatura, não se estabelece uma relação aparente entre as aplicações multilineares simétricas e os polinômios simétricos uma vez que o conceito de simetria ligado a estes dois entes matemáticos é de natureza distinta um do outro.

Uma propriedade relativa aos polinômios simétricos é que podemos substituir suas variáveis originais por outras variáveis convenientes; nesse sentido, introduzimos, agora, certos tipos de funções que são utilizadas para reescrever um polinômio simétrico:

Definição 3.20. Para $x=\left(x_{1}, \cdots, x_{m}\right) \in \mathbb{R}^{m}$, sejam:

$$
\begin{aligned}
\sigma_{1}(x) & =x_{1}+x_{2}+\cdots+x_{m} \\
\sigma_{2}(x) & =\sum_{i=1}^{m-1} \sum_{j=i+1}^{m} x_{i} x_{j} \\
\vdots & \\
\sigma_{m}(x) & =x_{1} x_{2} \cdots x_{m} .
\end{aligned}
$$

Estas funções $\sigma_{i}, i=1, \cdots, m$, que também são exemplos de polinômios homogêneos simétricos, são chamadas de funções simétricas elementares. 
O teorema seguinte nos garante que é sempre possível substituir as variáveis de um polinômio simétrico pelas variáveis $\sigma_{i}$ 's; antes de passarmos diretamente à sua demonstração, apresentamos o seguinte fato:

Seja $f: \mathbb{R} \longrightarrow \mathbb{R}$ um polinômio com raízes $x_{1}, x_{2}, \ldots, x_{m}$ e definido na variável $z$, dada por

$$
f(z)=\left(z-x_{1}\right) \cdot\left(z-x_{2}\right) \cdots\left(z-x_{m}\right)=z^{m}-\sigma_{1} z^{m-1}+\cdots+(-1)^{m} \sigma_{m}
$$

com $x_{1}, x_{2}, \cdots, x_{m} \in \mathbb{R}$; se tomarmos $x_{m}=0$ e, em seguida, dividirmos por $z$, obtemos:

$$
\left(z-x_{1}\right) \cdot\left(z-x_{2}\right) \cdots\left(z-x_{m-1}\right)=z^{m-1}-\left(\sigma_{1}\right)_{o} z^{m-2}+\cdots+(-1)^{m-1}\left(\sigma_{m-1}\right)_{o}
$$

sendo $\left(\sigma_{i}\right)_{o}$ a expressão de $\sigma_{i}$ para $x_{m}=0$. Logo, cada $\left(\sigma_{i}\right)_{o}$ é uma função simétrica elementar expressa nas primeiras $(m-1)$ variáveis.

Segue o teorema citado:

Teorema 3.21. (Teorema Fundamental das Funções Elementares): Todo polinômio simétrico $P: \mathbb{R}^{m} \longrightarrow \mathbb{R}$ de grau d pode ser escrito como

$$
P\left(x_{1}, \cdots, x_{m}\right)=Q\left(\sigma_{1}, \cdots, \sigma_{m}\right)
$$

com $\sigma_{i}, i=1,2, \cdots, m$ sendo as funções simétricas elementares e $Q$ um polinômio de grau $d, \forall(m, d) \in \mathbb{N} \times \mathbb{N}_{0}$.

Demonstração : Para a prova do teorema, vamos usar indução sobre $m$ :

- Para $m=1$, o teorema é verdadeiro pois, sendo $\sigma_{1}=x_{1}$, todo polinômio $P(x)$ é simétrico e $P\left(x_{1}\right)=P\left(\sigma_{1}\right)$.

- Assumimos que o teorema seja válido para polinômios definidos em até $(m-1)$ variáveis $(m>1)$.

- Vamos provar para $m$ variáveis; para tal, vamos usar indução sobre $d$ :

- Para $d=0$, o polinômio $P$ é constante em $m$ variáveis e o resultado é trivial.

- Suponhamos válido para todos os polinômios de grau $<d$ em $m$ variáveis; resta mostrar que vale para polinômios de grau $d$ em $m$ variáveis. 
- Seja dado $P: \mathbb{R}^{m} \longrightarrow \mathbb{R}$ um polinômio simétrico de grau $d$; tomando-se $x_{m}=0$, temos $P\left(x_{1}, x_{2}, \cdots, x_{m-1}, 0\right)=R\left(\left(\sigma_{1}\right)_{o},\left(\sigma_{2}\right)_{o}, \cdots,\left(\sigma_{m-1}\right)_{o}\right)$ para algum polinômio simétrico $R$ pois, neste caso, $R$ tem grau $\leq d$ em $(m-1)$ variáveis, sendo $\left(\sigma_{i}\right)_{0}, i=1, \ldots,(m-1)$ as funções simétricas elementares em $(m-1)$ variáveis. Poṛtanto, considerando-se o mesmo polinômio $R$ mas, agora, definido nas variáveis $\sigma_{1}, \sigma_{2}, \cdots, \sigma_{m-1}$, o polinômio $R\left(\sigma_{1}, \sigma_{2}, \cdots, \sigma_{m-1}\right)$ também tem grau $\leq d$.

Seja, agora, $S: \mathbb{R}^{m} \longrightarrow \mathbb{R}$ um polinômio definido por:

$$
S\left(x_{1}, x_{2}, \cdots, x_{m-1}, x_{m}\right)=P\left(x_{1}, x_{2}, \cdots, x_{m-1}, x_{m}\right)-R\left(\sigma_{1}, \sigma_{2}, \cdots, \sigma_{m-1}\right) .
$$

Com isso, $S$ é simétrico; como $P$ tem grau $d$ e $R$ tem grau $\leq d$ nas variáveis $\sigma_{1}, \sigma_{2}, \cdots, \sigma_{m-1}$ e, portanto, grau $\leq d$ nas variáveis $x_{1}, x_{2}, \cdots, x_{m-1}$, temos que $S$ tem grau $\leq d$. Além disso, para $x_{m}=0, \quad S\left(x_{1}, x_{2}, \cdots, x_{m-1}, 0\right)=0$. Logo, todos os termos de $S$ contêm $x_{m}$; como $S$ é simétrico, todos os termos também contêm $x_{1}, x_{2}, \cdots, x_{m-1}$.

Desta forma, fatorando todos os termos de $S$ por $x_{1} \cdot x_{2} \cdots x_{m-1} \cdot x_{m}=\sigma_{m}$, obtemos $S\left(x_{1}, x_{2}, \cdots, x_{m-1}, x_{m}\right)=\sigma_{m} \cdot g\left(x_{1}, x_{2}, \cdots, x_{m-1}, x_{m}\right)$, sendo $g$ um polinômio simétrico de grau $\leq d-m<d$. Se $d<m$, então $g \equiv 0$ e podemos escrever $P\left(x_{1}, \ldots, x_{m}\right)=$ $Q\left(\sigma_{1}, \sigma_{2}, \cdots, \sigma_{m}\right)=Q\left(\sigma_{1}, \sigma_{2}, \ldots, \sigma_{d}, 0,0, \ldots, 0\right)$ ficando o teorema, assim, demonstrado. Vamos supor que $d \geq m$; desta forma, por hipótese de indução sobre $d, g$ pode ser expresso em termos das funções simétricas elementares $\sigma_{1}, \sigma_{2}, \cdots, \sigma_{m}$ :

$$
g\left(x_{1}, x_{2}, \cdots, x_{m-1}, x_{m}\right)=\Psi\left(\sigma_{1}, \sigma_{2}, \cdots, \sigma_{m}\right)
$$

com $\Psi$ sendo um polinômio de grau $\leq d-m$.

Podemos, então, escrever $P$ como:

$$
\begin{aligned}
P\left(x_{1}, x_{2}, \cdots, x_{m-1}, x_{m}\right) & =S\left(x_{1}, x_{2}, \cdots, x_{m-1}, x_{m}\right)+R\left(\sigma_{1}, \sigma_{2}, \cdots, \sigma_{m-1}\right) \\
& =\sigma_{m} \cdot \Psi\left(\sigma_{1}, \sigma_{2}, \cdots, \sigma_{m}\right)+R\left(\sigma_{1}, \sigma_{2}, \cdots, \sigma_{m-1}\right) .
\end{aligned}
$$

Desta forma, o lado direito da última igualdade tem grau $\leq d$ e, por hipótese, $P$ tem grau $d$; logo, o lado direito tem grau $d$. 
Estamos interessados, na verdade, em escrever $P$ em termos das variáveis $\sum_{j=1}^{m} x_{j}^{i}$, $i=1,2, \cdots, m$, as potências semelhantes de $x_{i}$. O método de Newton, como apresentado em [11], garante que podemos reescrevê-lo nessas variáveis como um outro polinômio de mesmo grau usando fórmulas matemáticas simples que relacionam as variáveis $\sigma_{i} \mathrm{e} \sum_{j=1}^{m} x_{j}^{i}$. Isto pode ser visto através do seguinte corolário:

Corolário 3.22. Todo polinômio simétrico $P: \mathbb{R}^{m} \rightarrow \mathbb{R}$ de grau d pode ser escrito como

$$
P\left(x_{1}, x_{2}, \cdots, x_{m}\right)=q\left(s_{1}, s_{2}, \cdots, s_{m}\right)
$$

com $s_{i}=\sum_{j=1}^{m} x_{j}^{i}$ e qum polinômio de grau d.

Demonstração : Podemos relacionar todos os termos $s_{i}$ com as funções simétricas elementares $\sigma_{1}, \cdots, \sigma_{i}$ da seguinte maneira:

$$
s_{1}=\sigma_{1}, \quad s_{i}-s_{i-1} \cdot \sigma_{1}+s_{i-2} \cdot \sigma_{2}-\cdots+(-1)^{i-1} s_{1} \cdot \sigma_{i-1}+(-1)^{i} \cdot i \cdot \sigma_{i}=0, \quad \forall i \leq m
$$

e

$$
s_{i}-s_{i-1} \cdot \sigma_{1}+\cdots+(-1)^{m} s_{i-m} \cdot \sigma_{m}=0, \quad \forall i>m
$$

ou seja, podemos escrever de maneira única cada $\sigma_{i}$ em termos de $s_{1}, s_{2}, \cdots, s_{m}, \forall i$.

Logo, temos:

$$
P\left(x_{1}, x_{2}, \cdots, x_{m}\right)=Q\left(\sigma_{1}, \sigma_{2}, \cdots, \sigma_{m-1}\right)=q\left(s_{1}, s_{2}, \cdots, s_{m}\right)
$$

Esse resultado nos permite determinar a dimensão dos espaços vetoriais formados pelas raízes de polinômios simétricos reais $d$-homogêneos com $d$ ímpar:

Proposição 3.23. Seja $P: \mathbb{R}^{m} \longrightarrow \mathbb{R}$ um polinômio simétrico d-homogêneo com $d$ impar. Então, $P^{-1}(0)$ é $\left[\frac{m}{2}\right]$-lineável. 
Demonstração : Do exposto acima, podemos escrever,

$$
\forall x=\left(x_{1}, x_{2}, \cdots, x_{m}\right) \in \mathbb{R}^{m}, P\left(x_{1}, \cdots, x_{m}\right)=q\left(\sum_{j=1}^{m} x_{j}, \cdots, \sum_{j=1}^{m} x_{j}^{m}\right) .
$$

Em outras palavras, escrevemos $P(x)=\sum_{\alpha} a_{\alpha}\left(\sum_{j=1}^{m} x_{j}\right)^{\alpha_{1}} \cdots\left(\sum_{j=1}^{m} x_{j}^{m}\right)^{\alpha_{m}}$

sendo $a_{\alpha} \in \mathbb{R}$ e todas as parcelas de $P$ tomadas para todo $\alpha=\left(\alpha_{1}, \alpha_{2}, \cdots, \alpha_{m}\right)$ tais que $1 \alpha_{1}+2 \alpha_{2}+\cdots+m \alpha_{m}=d$. Importante ressaltar que, se $d \leq m$, então o polinômio $q$ é escrito apenas nas $d$ primeiras variáveis dessas potências semelhantes de $x_{i}$. Agora, para cada $\alpha$ fixado, como $d$ é ímpar, não pode ocorrer $\alpha_{j}=0, \forall j$ ímpar; portanto, pelo menos, um dos $\alpha_{j}$ 's deve ser ímpar para $j$ ímpar; desta forma, podemos concluir que cada parcela $P$ se anula nos vetores da forma $\left(e_{1}-e_{2}\right),\left(e_{3}-e_{4}\right), \cdots,\left(e_{2\left[\frac{m}{2}\right]-1}-e_{2\left[\frac{m}{2}\right]}\right)$ sendo $\left\{e_{i}: i=1,2, \cdots, m\right\}$ a base canônica de $\mathbb{R}^{m}$.

Logo, $\left[\left(e_{1}-e_{2}\right),\left(e_{3}-e_{4}\right), \cdots,\left(e_{2\left[\frac{m}{2}\right]-1}-e_{2\left[\frac{m}{2}\right]}\right)\right] \subset P^{-1}(0)$ e tem dimensão $\left[\frac{m}{2}\right]$.

\subsection{Polinômios 2-Homogêneos Definidos em Espaços de Banach de Dimensão Infinita}

Na seção anterior, apresentamos exemplos de polinômios homogêneos reais cujos conjuntos das raízes contêm espaços vetoriais de dimensão finita $n$ e, portanto, são n-lineáveis; porém, quando um polinômio $P$ está definido em um espaço de dimensão infinita, podemos, em alguns casos, mostrar que $P^{-1}(0)$ é lineável.

Esta seção tem como objetivo apresentar um resultado sobre os conjuntos das raízes dos polinômios 2-homogêneos que são lineáveis dependendo fundamentalmente do tipo de espaço em que estejam definidos. Este resultado se encontra no texto científico [2].

Para tal, necessitamos, primeiramente, dos seguintes lemas: 
Lema 3.24. (Lema de Zorn) : Seja X um conjunto parcialmente ordenado com a propriedade que cada subconjunto totalmente ordenado admite uma cota superior. Então, $X$ contém um elemento maximal.

Observação: Para definições de cada um dos entes matemáticos citados acima no lema, ver, por exemplo, [10], pg. 71 e 72.

Lema 3.25. Sejam $E$ um espaço normado de dimensão infinita e $V \subset E$ um subespaço de dimensão finita; então, para quaisquer $x, y \in E \backslash V$, existe uma curva contínua unindo $x$ a y e inteiramente contida em $E \backslash V$.

Demonstração : Sejam $x, y \in E \backslash V$. Consideremos $B:=\left\{v_{1}, v_{2}, \cdots, v_{n}\right\}$ uma base de $V$ e $X:=\left\{v_{1}, v_{2}, \cdots, v_{n}, x, y\right\}$ um conjunto de $E$. Assim, somente duas possibilidades devem ocorrer, a saber: $X$ é linearmente independente ou $X$ é linearmente dependente.

- Suponhamos que $X$ seja um conjunto linearmente independente. Seja $\phi:[0 ; 1] \longrightarrow E$ o segmento de reta unindo $x$ a $y$, ou seja, $\phi(t)=t x+(1-t) y, t \in[0 ; 1]$; vamos provar que $\phi([0 ; 1]) \subset E \backslash V$. Se existisse um vetor $v \in V$ tal que $v \in \phi([0 ; 1])$, então $v=\sum_{i=1}^{n} \alpha_{i} \cdot v_{i}=t_{0} \cdot x+\left(1-t_{0}\right) \cdot y$, com $\alpha_{i} \in \mathbb{R}, \forall i$ e $t_{0} \in[0 ; 1]$; logo, $\sum_{i=1}^{n} \alpha_{i} \cdot v_{i}-t_{0} \cdot x-\left(1-t_{0}\right) \cdot y=0$; pelo fato de $X$ ser linearmente independente, deveria ocorrer $t_{0}=0$ e $t_{0}=1$ (absurdo). Conseqüentemente, $\phi([0 ; 1]) \subset E \backslash V$ e $\phi$ é a função procurada.

- Suponhamos, agora, que o conjunto $X$, como definido anteriormente, seja linearmente dependente.; por hipótese, $\left\{v_{1}, v_{2}, \cdots, v_{n}, x\right\}$ e $\left\{v_{1}, v_{2}, \cdots, v_{n}, y\right\}$ são conjuntos linearmente independentes; como $\operatorname{dim} E=\infty$, existe um vetor $z \in E$ tal que $\left\{v_{1}, v_{2}, \cdots, v_{n}, x, z\right\}$ e $\left\{v_{1}, v_{2}, \cdots, v_{n}, y, z\right\}$ são conjuntos linearmente independentes. Consideremos os segmentos de reta $\phi_{1}:[0 ; 1] \longrightarrow E$ e $\phi_{2}:[0 ; 1] \longrightarrow E$ tais que $\phi_{1}(0)=x$, $\phi_{1}(1)=z, \phi_{2}(0)=z$ e $\phi_{2}(1)=y$. Assim, $\phi_{1}([0 ; 1]) \subset E \backslash V$ e $\phi_{2}([0 ; 1]) \subset E \backslash V$. Seja $\Psi:[0 ; 1] \longrightarrow E$ definida por

$$
\Psi(t)= \begin{cases}\phi_{1}(2 t)=2 t z+(1-2 t) x, & \text { se } 0 \leq t \leq \frac{1}{2} \\ 2 \phi_{2}(t)-y=2(1-t) z+(2 t-1) y, & \text { se } \frac{1}{2} \leq t \leq 1\end{cases}
$$


Como definida, $\Psi$ é uma curva contínua e $\Psi([0 ; 1]) \subset E \backslash V$. Assim, $\Psi$ é a função procurada.

Necessitamos, para a obtenção do principal resultado desta seção, da seguinte definição relativa às funções:

Definição 3.26. Uma função $f: E \longrightarrow \mathbb{R}$ é dita definida positiva se $f(x) \geq 0, \forall x \in E$ e $f(x)=0 \Longrightarrow x=0$.

A proposição seguinte, aparentemente natural e simples, mostra-nos que existem polinômios 2-homogêneos cujos conjuntos das raízes sejam n-lineáveis mas não lineáveis de acordo com a natureza do espaço de Banach real ao qual estes polinômios estão definidos.

Segue, então, a proposição:

Proposição 3.27. Seja E um espaço de Banach real; são equivalentes:

a) E admite um polinômio 2-homogêneo contínuo definido positivo.

b) Existe uma injeção contínua de E em um espaço de Hilbert.

c) Existe um polinômio 2-homogêneo $P$ definido em $E$ cujo conjunto das raízes está contido num subespaço de dimensão finita de $E$.

Demonstração : a) $\Rightarrow$ b) Seja $A$ a aplicação bilinear simétrica associada ao polinômio definido positivo 2-homogêneo $P$; seja $(E ;<,>)$ um espaço pré-Hilbert com produto interno definido como $\langle x, y\rangle=A(x, y), \forall x, y \in E$. Tomemos $H$ o espaço de Hilbert completado de $(E ;<,>)$ com a norma $\||\cdot|||$ proveniente do produto interno. Definimos a injeção $j: E \longrightarrow H$ como $j(x)=x$, que é contínua pois:

$$
\begin{aligned}
\left.\|j(x)\|\left\|^{2}=\right\|\|x\|\right|^{2} & =<x, x>=A(x, x)=P(x) \leq\|P\| \cdot\|x\|^{2} \Longrightarrow \\
& \Longrightarrow\|j(x)\|\|\leq \sqrt{\|P\|} \cdot\| x \|
\end{aligned}
$$


b) $\Rightarrow$ c) Por hipótese, existem $H$ (Hilbert) e $j: E \longrightarrow H$ uma injeção contínua tal que $j(x)=x$. Seja $A: E \times E \longrightarrow \mathbb{R}$ a aplicação bilinear simétrica definida por $A(x, y)=<j(x), j(y)>$; o polinômio 2-homogêneo $P: E \longrightarrow \mathbb{R}$ associado a $A$ é, então, dado por $P(x)=<j(x), j(x)>$. Logo:

$$
P(x)=0 \Longrightarrow<j(x), j(x)>=0 \Longrightarrow j(x)=0 \Longrightarrow x=0 \Longrightarrow P^{-1}(0)=0
$$

c) $\Rightarrow$ a) Vamos considerar que $\operatorname{dim} E=\infty$; para espaços $E$ de dimensão finita, a solução é trivial. Seja $P$ um polinômio 2-homogêneo cujo conjunto das raízes esteja contido num subespaço de dimensão finita $V \subset E$ com uma base $\left\{v_{1}, v_{2}, \cdots, v_{n}\right\}$. Primeiramente, observamos que $P(x)$ é sempre positivo ou sempre negativo para todo $x \in E \backslash V$ :

Se não, existiriam $x, y \in S_{E \backslash V}$, a esfera unitária de $E \backslash V$, tais que $P(x)<0<P(y)$. Agora, pelo lema 3.25, existe uma função $\phi:[0,1] \longrightarrow E \backslash V$ contínua tal que $\phi(0)=x$ e $\phi(1)=y$; então, teríamos $P \circ \phi(t)=0$ para algum $t \in[0 ; 1]$. Com isso, $\phi(t) \in V$ (absurdo pois $\phi(t) \in E \backslash V)$.

Desta forma, sem perda de generalidade, podemos assumir que $P(x)>0, \forall x \in E \backslash V$. Para a condição $P(x) \geq 0, \forall x \in E$ e $P(x)=0 \Longrightarrow x=0$, o próprio $P$ seria o polinômio procurado. Vamos considerar, agora, que $P$ não atenda essa última condição. A partir disso, vamos construir um polinômio 2-homogêneo $Q$ definido positivo em $E$. Como $V=\left[v_{1}, v_{2}, \cdots, v_{n}\right]$, então existem $g_{i} \in V^{*}, 1 \leq i \leq n \operatorname{com} g_{i}\left(v_{j}\right)=\delta_{i j}$. Pelo teorema de Hahn-Banach (ver, por exemplo, [16], pg. 221- 222), existem $f_{i} \in E^{*}$ tais que $\left.f_{i}\right|_{V}=g_{i}$, para todo $1 \leq i \leq n$. Consideremos $\pi: E \longrightarrow V$ dada por:

$$
\pi(x)=\sum_{i=1}^{n} f_{i}(x) v_{i}
$$

Desta forma, $\pi$ é uma projeção linear contínua e nos auxiliará a construir um polinômio definido positivo em $E$.

Como $\bar{B}_{V}$, a bola unitária fechada de $V$, é compacta, existe um $x_{0} \in \bar{B}_{V}$ tal que $P\left(x_{0}\right) \leq P(x), \forall x \in \bar{B}_{V} ;$ consideremos $P\left(x_{0}\right)=-\alpha$, com $\alpha \geq 0$. Como $\operatorname{dim} V<\infty$, as normas consideradas em $V$ são equivalentes; desta forma, existe $c>0$ tal que $\|x\| \leq \sqrt{c} \cdot\|x\|_{e}$, com $\|\cdot\|_{e}$ a norma euclidiana em $V$, para cada $x \in V$. Então, para cada 
$x=\sum_{i=1}^{n} f_{i}(x) \cdot v_{i}$, temos:

$$
\|x\|^{2} \leq c \cdot \sum_{i=1}^{n} f_{i}^{2}(x) \Longrightarrow \sum_{i=1}^{n} f_{i}^{2}(x) \geq \frac{\|x\|^{2}}{c}
$$

Consideremos, agora, o polinômio 2-homogêneo $Q$ definido por:

$$
Q(x)=P(x)+(\alpha+1) \cdot c \cdot \sum_{i=1}^{n} f_{i}^{2}(x)
$$

Então, $Q(x)>0, \forall x \in E \backslash V$. Vamos mostrar que $Q$ é positivo em $V$; consideremos arbitrariamente $x \in S_{V}$, a esfera unitária de $V$; então, $\|x\|=1$ e, da inequação 3.1, temos $\sum_{i=1}^{n} f_{i}^{2}(x) \geq \frac{1}{c}$. Com isso, $\forall x \in S_{V}$, temos:

$Q(x) \geq-\alpha+(\alpha+1) \cdot c \cdot \sum_{i=1}^{n} f_{i}^{2}(x) \Longrightarrow Q(x) \geq 1, \forall x \in S_{V}$. Por outro lado, sabemos que $Q(\lambda x)=\lambda^{2} Q(x)$, para $\forall x \in E, \forall \lambda \in \mathbb{R}$; com isso, $Q(x) \geq 0, \forall x \in V$. Portanto, $Q(x) \geq 0, \forall x \in E$. Além disso, se existisse $x \in V$ não nulo tal que $Q(x)=0$, então poderíamos tomá-lo, sem perda de generalidade, em $S_{V}$; porém isso não pode ocorrer pois sabemos que $Q(x) \geq 1, \forall x \in S_{V}$.

Logo, $Q(x)=0 \Longrightarrow x=0$ e $Q$ é um polinômio 2-homogêneo definido positivo.

Finalmente, segue o principal resultado desta seção:

Teorema 3.28. Seja E um espaço de Banach real que não admite um polinômio 2-homogêneo definido positivo. Então, para cada $P \in P\left({ }^{2} E\right)$, existe um subespaço $S_{0}$ de $E$ de dimensão infinita $P_{\left.\right|_{S_{0}}} \equiv 0$.

Demonstração : Suponhamos que $E$ não admita um polinômio 2-homogêneo definido positivo e tomemos $P \in P\left({ }^{2} E\right)$ arbitrário. Seja $\mathcal{S}=\{S \subset E: \mathrm{S}$ é subespaço de E e $\left.P_{I_{S}} \equiv 0\right\} ; \mathcal{S}$ não possui somente $\{0\}$ como elemento pois, como $E$ não admite um polinômio 2-homogêneo definido positivo, para $P \in P\left({ }^{2} E\right)$, existe $x \in E \backslash\{0\}$ tal que 
$P(x) \leq 0$. Se $P(x)=0$, então o espaço $[x] \in \mathcal{S}$; se $P(x)<0$, então, podemos tomar $y \in E$ tal que $\{x, y\}$ seja linearmente independente e $P(y) \geq 0$ (existe esse $y$ pois, caso contrário, o conjunto das raízes de $P$ teria dimensão finita e, pela proposição anterior, $E$ admitiria um polinômio 2-homogêneo definido positivo - absurdo). O segmento que une $x$ a $y$ possui um elemento $z \neq 0$ tal que $P(z)=0$. Nesse caso, $[z] \in \mathcal{S}$.

Como foi definido, $\mathcal{S}$ é parcialmente ordenado por inclusão. Seja $\mathcal{D}=\left\{S_{\alpha}\right\}_{\alpha \in \Lambda}$ ( $\Lambda$ um conjunto de índices) um subconjunto totalmente ordenado de $\mathcal{S}$; então $\bigcup_{\alpha \in \Lambda} S_{\alpha}$ é cota superior para $\mathcal{D}$. Logo, pelo Lema de Zorn, $\mathcal{S}$ possui um elemento maximal; denotamos esse elemento por $S_{0}$. Vamos mostrar que $\operatorname{dim} S_{0}=\infty$ :

Suponhamos que $\operatorname{dim} S_{0}=n$ para algum $n \in \mathbb{N}$ e que $B=\left\{v_{1}, \cdots, v_{n}\right\}$ seja uma base de $S_{0}$. Seja $A: E \times E \longrightarrow \mathbb{R}$ a aplicação bilinear simétrica associada a $P$. Consideremos $T=\bigcap_{x \in S_{0}} \operatorname{ker} A_{x} \operatorname{com} A_{x}: E \longrightarrow \mathbb{R}$ funcionais lineares dados por $A_{x}(y)=A(x, y)$.

Afirmamos que $S_{0} \subset T$. De fato, seja $y \in S_{0}$ fixado. Então, $\forall x \in S_{0}, x+y \in S_{0}\left(S_{0}\right.$ é subespaço); logo:

$$
\begin{aligned}
& 0=P(x+y)=A(x+y, x+y)=A(x, x)+A(x, y)+A(y, x)+A(y, y)= \\
& 0+2 A(x, y)+0=2 A_{x}(y) \Longrightarrow A_{x}(y)=0, \forall x \in S_{0} \Longrightarrow y \in \operatorname{ker} A_{x}, \forall x \in S_{0},
\end{aligned}
$$

isto é, $y \in T$ e, conseqüentemente, $S_{0} \subset T$. Notemos que, para todo $x \in S_{0}$, se $y \in T$ e $P(y)=0$, então, para quaisquer escalares $\lambda_{1}$ e $\lambda_{2}$, temos $P\left(\lambda_{1} x+\lambda_{2} y\right)=\lambda_{1}^{2} P(x)+$ $2 \lambda_{1} \lambda_{2} A_{x}(y)+\lambda_{2}^{2} P(y)=0$. Logo, $y$ pertence a um subespaço de $\mathcal{S}$. Com isso, pela maximalidade de $S_{0}, y \in S_{0}$.

Afirmamos que $S_{0}$ é complementado em $T$; de fato, seja $B^{*}=\left\{g_{1}, \ldots, g_{n}\right\}$ a base dual da base $B$ de $S_{0}$. Então, pelo teorema de Hahn-Banach, existem $f_{i} \in T^{*}$ tais que $\left.f_{i}\right|_{S_{0}}=g_{i}, i=1, \ldots, n$. Tomando-se a função $\pi: T \longrightarrow S_{0} \operatorname{com} \pi(x)=\sum_{i=1}^{n} f_{i}(x) \cdot v_{i}$, temos que $\pi$ é uma projeção linear contínua e, conseqüentemente, $S_{0}$ é complementado em $T$.

Seja, então, o subespaço $Y$ de $E$ tal que $T=S_{0} \oplus Y$. Como todas as raízes de $P_{\left.\right|_{T}}$ estão em $S_{0}$, ou $P_{\left.\right|_{T}}$ ou $-P_{\left.\right|_{T}}$ é positivo em $Y$. Suponhamos, então, sem perda de generalidade, que $P_{l_{T}}$ é positivo em $Y$. Como $\operatorname{dim} S_{0}=n$, o seguinte polinômio 2-homogêneo é definido 
positivo em $T$ :

$$
Q(x)=P_{\left.\right|_{T}}(x)+\sum_{i=1}^{n} f_{i}^{2}(x)
$$

No entanto, queremos mostrar que a hipótese assumida de $S_{0}$ ter dimensão finita é absurda; para isso, vamos exibir um polinômio positivo definido 2-homogêneo contínuo definido em $E$ contrariando a hipótese inicial de $E$ não admitir tal polinômio. Nesse sentido, vamos mostrar que $T$ é complementado em $E$ da seguinte maneira:

Observemos que $T=\bigcap_{i=1}^{n} k e r A_{v_{i}}$ pois $\bigcap_{x \in S_{0}} \operatorname{ker} A_{x} \subseteq \bigcap_{i=1}^{n} k e r A_{v_{i}}$ é trivial e, para cada $x_{0} \in \bigcap_{i=1}^{n} \operatorname{ker} A_{v_{i}}$, temos que $A\left(v_{i} ; x_{0}\right)=0, \forall i \Longrightarrow \forall \beta_{i} \in \mathbb{R}, A\left(\sum_{i=1}^{n} \beta_{i} v_{i} ; x_{0}\right)=0 \Longrightarrow$ $A\left(x ; x_{0}\right)=0, \forall x \in S_{0}$, isto é, $A_{x}\left(x_{0}\right)=0, \forall x \in S_{0}$ e, conseqüentemente, $x_{0} \in \bigcap_{x \in S_{0}} k e r A_{x}$.

O subespaço $T$ assim definido tem codimensão finita em $E$ pois, considerando a aplicação linear $\phi: E \longrightarrow \mathbb{R}^{n}$ definida por:

$$
\phi(x)=\left(A_{v_{1}}(x), A_{v_{2}}(x), \cdots, A_{v_{n}}(x)\right)
$$

temos ker $\phi=\bigcap_{i=1}^{n} \operatorname{ker} A_{v_{i}}=T$. Então, $\phi$ determina um isomorfismo $\psi: E / T \longrightarrow \operatorname{Im} \phi \subseteq \mathbb{R}^{n}$. Com isso, $E / T$ tem dimensão $\leq n$.

Assim, $E / T$ tem dimensão finita, isto é, $T$ tem codimensão finita em $E$ e, sendo fechado, segue que $T$ é complementado em $E$.

Finalmente, tomando-se $\pi_{T}: E \longrightarrow T$ uma projeção linear contínua, podemos definir o seguinte polinômio 2-homogêneo:

$$
Q_{1}(x)=Q \circ \pi_{T}(x)+\sum_{i=1}^{n} A_{v_{i}}^{2}(x)
$$

sendo $Q$ como definido na equação 3.2. Desta forma, podemos reescrever esse polinômio definido em $E$ como

$$
Q_{1}(x)= \begin{cases}Q(x), & \text { se } x \in T \\ \sum_{i=1}^{n} A_{v_{i}}^{2}(x), & \text { se } x \in E \backslash T\end{cases}
$$


Portanto, a demonstração está concluída uma vez que $Q_{1}$ é um polinômio 2-homogêneo definido positivo contínuo.

Podemos afirmar, através do seguinte exemplo, que se um espaço de Banach é separável, então admite um polinômio 2-homogêneo definido positivo.

Exemplo 3.29. Seja $E$ um espaço de Banach real separável com $\left\{x_{n}\right\}_{n \in \mathbb{N}}$ um subconjunto denso da bola unitária $B_{E}$. Para cada $n$, seja $\phi_{n} \in E^{*}$ com $\left\|\phi_{n}\right\|=1$ e $\phi_{n}\left(x_{n}\right)=1$. Definimos o polinômio 2-homogêneo $P: E \longrightarrow \mathbb{R}$ por:

$$
P(x)=\sum_{n=1}^{\infty} \frac{\phi_{n}^{2}(x)}{2^{n}}
$$

Se $P(y)=0$ para algum $y \in E \backslash\{0\}$, então $P(x)=0$ para o vetor unitário $x=\frac{y}{\|y\|}$. Isto significa que $\phi_{n}(x)=0, \forall n \in \mathbb{N}$. Como $\left\{x_{n}\right\}_{n}$ é denso em $B_{E}$, existem valores de $n \in \mathbb{N}$ tais que $\left\|x-x_{n}\right\|<1$. Mas, para tais $n$, segue

$$
\left|\phi_{n}\left(x-x_{n}\right)\right|=\left|\phi_{n}(x)-\phi_{n}\left(x_{n}\right)\right|=1 \leq\left\|\phi_{n}\right\| \cdot\left\|x-x_{n}\right\|<1
$$

(absurdo).

Observação: Nem todo espaço não enumerável satisfaz o teorema 3.28; por exemplo, usando argumentação semelhante ao exposto no exemplo para o espaço $l_{\infty}$, verifica-se que este espaço admite um polinômio 2-homogêneo contínuo definido positivo (ver, por exemplo, [2], pg. 13). Também em [2], o autor afirma que os espaços $c_{0}(\Gamma)$ e $l_{p}(\Gamma), p>2$, com $\Gamma$ um conjunto não enumerável, atendem ao teorema 3.28. Com relação ao subespaço $S_{0}$ definido no mesmo teorema, uma questão natural é se $S_{0}$ é também não separável; para os espaços $l_{p}(\Gamma), p>4$, a resposta é afirmativa (ver, por exemplo, [2], pg. 14). 


\section{Referências Bibliográficas}

[1] Z. Abud e R. Alencar Contagens e escolhas na teoria dos conjuntos, IME USP, (2003)

[2] R. M. Aron, An introduction to polynomials on Banach spaces,, Extracta Math. 17 (2002). $n^{\circ} 3,303-329$.

[3] R. M. Aron, R. Gonzalo and A. Zagorodnyuk, Zeros of real polynomials, Linear and Multilinear Algebra 48 (2000), $n^{\circ}$ 2, 107-115.

[4] R.M. Aron, V. I. Guraryi and J. B. Seoane, Lineability and spaceability of sets of functions on $\mathbb{R}$, Proc. Amer. Math Soc. 133 (2005), $n^{\circ}$ 3, 795-803.

[5] R. P. Boas Jr., A Primer of Real Functions,, The Carus Mathematical Monographs, $n^{o} 13$, John Wiley and Sons, Inc., New York, 1960.

[6] C. B. Boyer, História da Matemática, Edgard Blücher Ltda., Programa Nacional Biblioteca do Professor, MEC-FAE, 1994.

[7] A. M. Bruckner, Differentiation of Real Functions, Lectures Notes in Math., vol. 659 Springer-Verlag, Berlin, 1978.

[8] A. M. Bruckner and J. L. Leonard, Derivatives, Amer. Math. Monthly 73 (1966), $n^{\circ} 4$, part II, 24-56.

[9] K. Ciesielski, Set Theory for the Working Mathematician, London Mathematical Society Student Texts, vol. 39, Cambridge University Press, Cambridge, 1997.

[10] F. U. Coelho e M. L. Lourenço, Um Curso de Álgebra Linear, Editora da Universidac de São Paulo, 2001. 
[11] P. Dubreil. A'gebre. Tome I: Équir'alences: Opéraions, Groupes. Anneaux, Corps. Troisième édition revue et augmentée. Préface de Gaston Julia. Cahiers Scientifiques; Fasc. XX, Gautier-Villars \& cie.. Éditeur-Imprimeur-Libraire, Paris. 1963.

[12] J. A. Gatica, Introducción a la Integral de Lebesgue en la Recta. Programa Regional de Desarrollo Científico y Tecnologico. Departamento de Asuntos Científicos, Secretaría General de la Organización de los Estados Americanos, Washington. D.C..1977.

[13] B. R. Gelbaum and J. .I. H. Olmsted, Counterexamples in Analysis, The Mathesis Series, Holden-Day Inc., San Francisco, Calif., 1964.

[14] E. W. Hobson, Theory of Functions of Real Variable II, Dover, New York, 1957.

[15] Y. Katznelson and K. Stromberg, Everywhere differentiable, nowhere monotone functions, Amer. Math. Monthly 81 (1974). 349-354.

[16] E. Kreysig, Introductory Functional Analysis with Applications, John Wiley \& Sons, New York-London-Sydney, 1978.

[17] H. Lebesgue, Leçons sur l'Integration, Gautier-Villars 1904.

[18] E. L. Lima, Curso de Análise Vol. 1, Projeto Euclides, Instituto de Matemática Pura e Aplicada, Rio de Janeiro, 2000.

[19] J. Mujica, Complex analysis in Banach spaces, Math. Studies 120 (1986), North-Holland, Amsterdan.

[20] L. Pelegrini. Lm Teorema de Hahn-Banach para polinòmios homogênєos, Dissertação de Mestrado, IME-USP, (2001). 